\title{
CHANDRA OBSERVATIONS OF THE HIGHEST REDSHIFT QUASARS FROM THE SLOAN DIGITAL SKY SURVEY
}

\author{
Ohad Shemmer, ${ }^{1}$ W. N. Brandt, ${ }^{1}$ Donald P. Schneider, ${ }^{1}$ Xiaohui Fan, ${ }^{2}$ Michael A. Strauss, ${ }^{3}$ \\ Aleksandar M. Diamond-Stanic, ${ }^{2}$ Gordon T. Richards, ${ }^{3}$ Scott F. Anderson, ${ }^{4}$ \\ James E. GunN, ${ }^{3}$ and Jon BRINKMANN ${ }^{5}$ \\ Received 2006 January 6; accepted 2006 February 17
}

\begin{abstract}
We present new Chandra observations of $21 z>4$ quasars, including 11 sources at $z>5$. These observations double the number of X-ray-detected quasars at $z>5$, allowing investigation of the X-ray spectral properties of a substantial sample of quasars at the dawn of the modern universe. By jointly fitting the spectra of $15 z>5$ radio-quiet quasars (RQQs), including sources from the Chandra archive, with a total of 185 photons, we find a mean X-ray powerlaw photon index of $\Gamma=1.95_{-0.26}^{+0.30}$, and a mean neutral intrinsic absorption column density of $N_{\mathrm{H}} \lesssim 6 \times 10^{22} \mathrm{~cm}^{-2}$. These results show that quasar X-ray spectral properties have not evolved up to the highest observable redshifts. We also find that the mean optical-X-ray spectral slope $\left(\alpha_{\mathrm{ox}}\right)$ of optically selected $z>5 \mathrm{RQQs}$, excluding broad absorption line quasars, is $\alpha_{\mathrm{ox}}=-1.69 \pm 0.03$, which is consistent with the value predicted from the observed relationship between $\alpha_{\mathrm{ox}}$ and ultraviolet luminosity. Four of the sources in our sample are members of the rare class of weak emission-line quasars, and we detect two of them in X-rays. We discuss the implications our X-ray observations have for the nature of these mysterious sources and, in particular, whether their weak-line spectra are a consequence of continuum boosting or a deficit of high-ionization line-emitting gas.
\end{abstract}

Subject headings: galaxies: active — galaxies: nuclei — quasars: emission lines — quasars: general — X-rays: galaxies

Online material: color figure

\section{INTRODUCTION}

During the past 5 years over 100 quasars at $z>4$ have been detected in X-rays, ${ }^{6}$ probing the inner regions of active galactic nuclei (AGNs) when the universe was $\$ 1.5$ Gyr old (e.g., Kaspi et al. 2000; Brandt et al. 2002; Bechtold et al. 2003; Vignali et al. 2003a, 2003b, 2005; Shemmer et al. 2005). Those studies have provided measurements of the $\mathrm{X}$-ray power-law photon index, intrinsic absorption column density, and the optical-X-ray spectral energy distribution (SED) of a few $z>4$ quasars that are particularly bright in X-rays, as well as the mean properties of large samples of these sources via joint fitting of their X-ray spectra (e.g., Page et al. 2005; Shemmer et al. 2005; Vignali et al. 2003a, $2003 \mathrm{~b}, 2005)$. The results demonstrated that the $\mathrm{X}$-ray spectra of $z>4$ quasars do not differ significantly from those of AGNs at lower redshift, and that the X-ray photon index of AGNs does not depend on luminosity (but see also Bechtold et al. 2003; Dai et al. 2004; Grupe et al. 2006). This is generally consistent with recent observations showing that the longer wavelength SEDs of quasars have not significantly evolved over cosmic time; e.g., see Stern et al. (2000), Carilli et al. (2001), and Petric et al. (2003) for radio observations, and Schneider et al. (1991), Vanden Berk et al. (2001), and Pentericci et al. (2003) for rest-frame optical-ultraviolet (UV) observations. While the X-ray spectral

\footnotetext{
${ }^{1}$ Department of Astronomy and Astrophysics, Pennsylvania State University, University Park, PA 16802; ohad@astro.psu.edu.

2 Steward Observatory, University of Arizona, 933 North Cherry Avenue, Tucson, AZ 85721

3 Princeton University Observatory, Peyton Hall, Princeton, NJ 08544.

4 Department of Astronomy, University of Washington, Box 351580, Seattle, WA 98195.

5 Apache Point Observatory, P.O. Box 59, Sunspot, NM 88349-0059.

${ }^{6}$ See http://www.astro.psu.edu/users/niel/papers/highz-xray-detected.dat for a regularly updated compilation of X-ray detections at $z>4$.
}

properties of $4<z<5$ quasars are now fairly well established, the situation for quasars at the highest observable redshifts, $z>5$, is not yet as clear. Only loose constraints on the typical X-ray spectral properties of $z>5$ quasars are available, mainly due to the small number of such sources discovered so far $(\lesssim 50)$. Obtaining X-ray spectral information for the highest redshift quasars is important for understanding the physical processes and environments of AGNs at the end of the reionization epoch (e.g., Loeb \& Barkana 2001).

$\mathrm{X}$-ray observations of $z>5$ quasars are challenging for present-day X-ray missions such as the Chandra X-Ray Observatory and $X M M$-Newton. Typically, exposure times of $\approx 10 \mathrm{ks}$ or more are required to detect $\sim 5$ photons from these sources, and $\gtrsim 100 \mathrm{ks}$ is required to obtain a reliable measurement of the photon index (Farrah et al. 2004; Schwartz \& Virani 2004). Prior to this work, only 11 quasars (10 of which are radio quiet, and one that is radio loud) at $z>5$ had been X-ray-detected (Brandt et al. 2001, 2002; Vignali et al. 2001, 2002, 2003b; Barger et al. 2002; Mathur et al. 2002; Bechtold et al. 2003; Steffen et al. 2004). In this work we add 11 more X-ray detections of $z>5$ quasars ${ }^{7}$ and create a statistically meaningful sample to investigate the mean X-ray properties of these sources. In addition, we present new X-ray observations of four $z \sim 4.5$ sources that belong to the rare class of weak (or absent) emission-line quasars (hereafter WLQs); only about a dozen sources of this type have been discovered at $z>2$ (e.g., Fan et al. 1999; Anderson et al. 2001; Collinge et al. 2005; Schneider et al. 2005).

This paper is organized as follows. In $\S 2$ we describe the Chandra observations and their reduction; the results are presented

\footnotetext{
7 The X-ray detections of two of these sources, SDSS J1053+5804 and SDSS J2228-0757, were reported briefly by Schneider et al. (2005); here we provide a full description of their detections (see $\S 3$ ).
} 


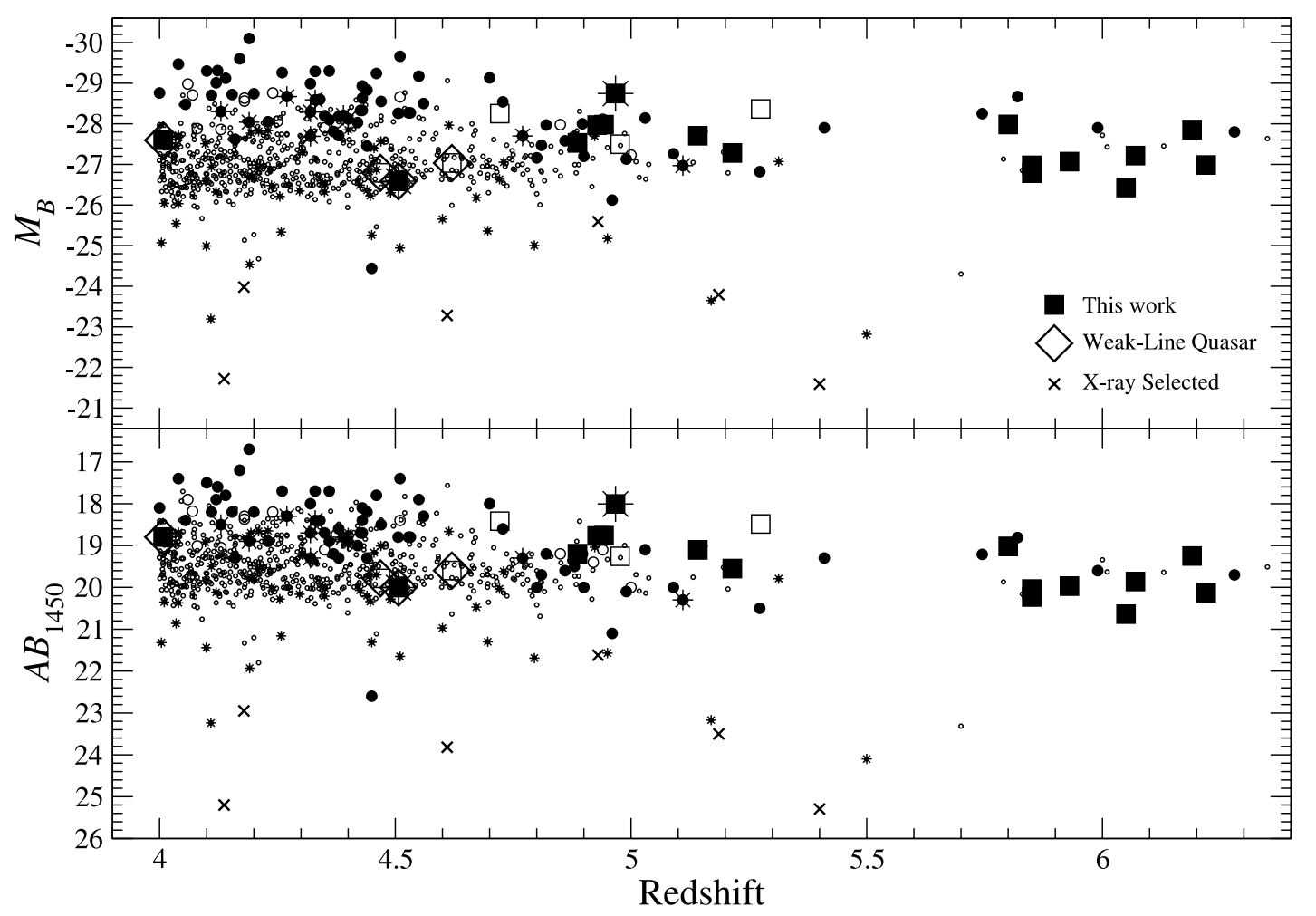

FIG. 1. $-M_{B}$ (top) and $A B_{1450}$ (bottom) vs. redshift for all published $z>4$ quasars (data were compiled from this work; Bassett et al. 2004; Steffen et al. 2004; Mahabal et al. 2005; Vignali et al. 2005; Fan et al. 2006). Large symbols indicate X-ray-targeted quasars. Open symbols indicate targeted quasars that are X-rayundetected, but have meaningful upper limits (i.e., $\alpha_{\mathrm{ox}}<-1.50$ ). Stars indicate RLQs and radio-moderate quasars. Squares indicate sources from this work, and enclosing diamonds indicate the WLQs in our sample. Note that two other $z>4$ WLQs, SDSS J0040-0915 (Schneider et al. 2003) and SDSS J133550.81+353315.8 (Fan et al. 2006), are not marked with an enclosing diamond, for clarity. [See the electronic edition of the Journal for a color version of this figure.]

in $\S 3 . \operatorname{In} \S 4$ we provide notes on individual interesting sources in our sample. In $\S 5$ we discuss the mean X-ray spectral properties of $z>5$ quasars. In $\S 6$ we discuss the results of our X-ray observations of WLQs, and how they may shed light on the nature of these sources. A summary of our main results is given in $\S 7$. Complete quasar names are given in the tables and in the headers of $\S 4$, and their abbreviated versions are used throughout the text. Luminosity distances in the paper are determined using the standard cosmological model with parameters $\Omega_{\Lambda}=0.7$, $\Omega_{M}=0.3$, and $H_{0}=70 \mathrm{~km} \mathrm{~s}^{-1} \mathrm{Mpc}^{-1}$ (e.g., Spergel et al. 2003).

\section{SAMPLE SELECTION, OBSERVATIONS, AND DATA REDUCTION}

We have targeted some of the optically brightest quasars at $z \gtrsim 5$ (see Fig. 1) to investigate quasar X-ray spectral properties and optical-X-ray SEDs at the highest observable redshifts. Targets with $z \lesssim 5.4$ were mostly selected from the Sloan Digital Sky Survey (SDSS; York et al. 2000) Data Release 3 (DR3) quasar catalog (Schneider et al. 2005). Quasars at higher redshifts cannot be detected with the SDSS quasar-selection algorithm (Richards et al. 2002), since Ly $\alpha$ shifts from the $i$ band to the $z$ band, and detections require the aid of near-infrared imaging and spectroscopy (e.g., Fan et al. 2004). We obtained short ( 3-30 ks) X-ray observations of 19 of the most distant quasars known with Chandra during Cycles 4 and 6 (see Fig. 1). The observation log appears in Table 1, where we also list references to the quasars' discovery papers. The targets include 18 newly discovered quasars from the SDSS survey (12 of which appear in the DR3 catalog; Schneider et al. 2005) and one previously X-ray-targeted quasar, SDSS J1532-0039, at $z=4.62$, which is the prototype of the WLQ class (Fan et al. 1999; Vignali et al. 2001). Three other WLQs from DR3 are included in our sample; our aim is to utilize the X-ray data to shed light on the nature of these sources. Our sample includes one radio-loud quasar (RLQ), SDSS J0011+1446, and two broad absorption line quasars (BALQSOs), SDSS J1048+4637 and SDSS J1653+ 4054. The Cycle 4 and 6 sources were observed with the Advanced CCD Imaging Spectrometer (ACIS; Garmire et al. 2003) with the S3 CCD at the aim point. In addition, we add to our sample serendipitous Chandra detections of two $z>4$ quasars, SDSS J1026+4719 at $z=4.94$ from Cycle 1, and SDSS J1053+ 5804 at $z=5.21$ from Cycle 2 (see Table 1); both of these sources were observed with ACIS-I and lie near the edge of the field of view.

Faint mode was used for the event telemetry format in all the observations, and ASCA (Advanced Satellite for Cosmology and Astrophysics) grade 0, 2, 3, 4, and 6 events were used in the analysis, which was carried out using standard CIAO ${ }^{8}$ version 3.2 routines. No background flares are present in these observations. Source detection was carried out with wavdetect (Freeman et al. 2002) using wavelet transforms (with wavelet scale sizes of $1,1.4,2,2.8$, and 4 pixels) and a false-positive probability threshold of $10^{-4}$. Given the small number of pixels being searched due to the accurate a priori source positions and the subarcsecond onaxis angular resolution of Chandra, the probability of spurious detections is extremely low; most of the sources were in fact detected at a false-positive probability threshold of $10^{-6}$.

The X-ray counts detected in the ultrasoft band $(0.3-0.5 \mathrm{keV})$, the soft band $(0.5-2 \mathrm{keV})$, the hard band $(2-8 \mathrm{keV})$, and the full

\footnotetext{
${ }^{8}$ Chandra Interactive Analysis of Observations. See http://asc.harvard.edu/
} 
TABLE 1

Chandra OBSERvations Log

\begin{tabular}{|c|c|c|c|c|c|c|c|}
\hline $\begin{array}{l}\text { Object } \\
(\text { SDSS J) }\end{array}$ & $z$ & $\begin{array}{c}\Delta_{\mathrm{ox}}{ }^{\mathrm{a}} \\
(\operatorname{arcsec})\end{array}$ & X-Ray Observation Date & Chandra Cycle No. & $\begin{array}{c}\text { Exposure Time }{ }^{\mathrm{b}} \\
(\mathrm{ks})\end{array}$ & Reference & Notes \\
\hline $000239.39+255034.8 \ldots \ldots \ldots \ldots . . . .$. & 5.80 & 0.3 & 2005 Jan 24 & 6 & 5.87 & 1 & \\
\hline $001115.23+144601.8 \ldots \ldots \ldots \ldots \ldots . .$. & 4.97 & 0.3 & 2003 May 20 & 4 & 3.49 & 2 & \\
\hline $084035.09+562419.9 \ldots \ldots \ldots \ldots \ldots$ & 5.85 & 0.2 & 2005 Feb 3-4 & 6 & 15.84 & 3 & \\
\hline $102622.89+471907.0 \ldots \ldots \ldots \ldots . .$. & 4.94 & 1.6 & 2000 June $7-8$ & 1 & $2.29^{\mathrm{e}}$ & 2 & Off-axis on ACIS-I \\
\hline $130216.13+003032.1 \ldots \ldots \ldots \ldots \ldots$ & 4.47 & $\ldots$ & 2003 May 17 & 4 & 10.81 & 2 & Weak emission lines \\
\hline $140850.91+020522.7 \ldots \ldots \ldots \ldots \ldots$ & 4.01 & 0.5 & 2005 Mar 2 & 6 & 6.00 & 2 & Weak emission lines \\
\hline $141111.29+121737.4 \ldots \ldots \ldots \ldots \ldots$ & 5.93 & 0.2 & 2005 Mar 17 & 6 & 14.27 & 1 & \\
\hline $143352.20+022713.9 \ldots \ldots \ldots \ldots$ & 4.72 & $\ldots$ & 2003 Apr 20 & 4 & 3.50 & 2 & \\
\hline $144231.72+011055.2 \ldots \ldots \ldots \ldots . .$. & 4.51 & 0.3 & 2002 Dec 16 & 4 & 11.01 & 2 & Weak emission lines \\
\hline $153259.96-003944.1^{\mathrm{c}} \ldots \ldots \ldots \ldots \ldots$ & 4.62 & $\ldots$ & 2005 Apr 2 & 6 & 9.83 & 5 & Weak emission lines \\
\hline $153650.26+500810.3 \ldots \ldots \ldots \ldots \ldots$ & 4.93 & 0.2 & 2005 Sep 23 & 6 & 4.62 & 2 & \\
\hline $165354.61+405402.1 \ldots \ldots \ldots \ldots . .$. & 4.98 & $\ldots$ & 2002 Dec $8-9$ & 4 & 3.12 & 2 & Broad absorption line quasar \\
\hline $222509.19-001406.8 \ldots \ldots \ldots \ldots . .$. & 4.89 & 0.4 & 2003 Sep $10-11$ & 4 & 3.45 & 2 & \\
\hline $222845.14-075755.3 \ldots \ldots \ldots \ldots . .$. & 5.14 & 0.7 & 2003 May 5 & 4 & 7.04 & 2 & \\
\hline
\end{tabular}

Notes.-The optical positions of the quasars have been obtained from the reference given in the seventh column, and the X-ray positions have been obtained with wavdetect.

${ }^{a}$ Distance between the optical and X-ray positions; missing values indicate no X-ray detection.

b The Chandra exposure time has been corrected for detector dead time.

c An additional $5.09 \mathrm{ks}$ Chandra exposure of the source is included in the current analysis, raising the total exposure time to $14.92 \mathrm{ks}$ (see Vignali et al. 2001 and $\S 2$ for details).

d The optical coordinates of this source from Fan et al. (2004) have been corrected using the SDSS DR4 astrometry.

e Actual exposure time, not corrected by the exposure map.

References.- (1) Fan et al. (2004); (2) Schneider et al. (2005); (3) Fan et al. (2006); (4) Fan et al. (2003); (5) Fan et al. (1999).

TABLE 2

X-Ray Counts, Band Ratios, and Effective Photon Indices

\begin{tabular}{|c|c|c|c|c|c|c|}
\hline \multirow{2}{*}{$\begin{array}{l}\text { ОвЈест } \\
\text { (SDSS J) }\end{array}$} & \multicolumn{4}{|c|}{ X-RAY Counts ${ }^{\mathrm{a}}$} & \multirow[b]{2}{*}{ BAND RATIO $^{\mathrm{b}}$} & \multirow[b]{2}{*}{$\Gamma^{\mathrm{b}}$} \\
\hline & $0.3-0.5 \mathrm{keV}$ & $0.5-2 \mathrm{keV}$ & $2-8 \mathrm{keV}$ & $0.5-8 \mathrm{keV}$ & & \\
\hline $000239.39+255034.8 \ldots \ldots \ldots \ldots \ldots \ldots \ldots \ldots \ldots \ldots$ & $<4.8$ & $5.0_{-2.2}^{+3.4}$ & $<3.0$ & $5.0_{-2.2}^{+3.4}$ & $<0.60$ & $>1.2$ \\
\hline $000552.34-000655.8 \ldots \ldots \ldots \ldots \ldots \ldots \ldots \ldots \ldots \ldots \ldots$ & $<3.0$ & $16.4_{-4.0}^{+5.2 .}$ & $<3.0$ & $17.6_{-4.1}^{+5.2}$ & $<0.18$ & $>2.2$ \\
\hline $001115.23+144601.8 \ldots \ldots \ldots \ldots \ldots \ldots \ldots \ldots \ldots \ldots \ldots$ & $6.9_{-2.6}^{+3.8}$ & $98.1_{-9.9}^{+10.9}$ & $28.8_{-5.3}^{+6.4}$ & $126.9_{-11.2}^{+12.3}$ & $0.29_{-0.06}^{+0.07}$ & $1.7_{-0.2}^{+0.2}$ \\
\hline $084035.09+562419.9$ & $<3.0$ & $\begin{array}{r}-9.9 \\
3.0_{-1.6}^{+2.9}\end{array}$ & $<4.8$ & $3.0_{-1.6}^{+2.2}$ & $<1.60$ & $>0.3$ \\
\hline $102622.89+471907.0$ & $<3.0$ & $3.7_{-1.8}^{-1.6}$ & $<6.4$ & $5.3_{-2.2}^{+3.6}$ & $<1.73$ & $>0.2$ \\
\hline $104845.05+463718.3$ & $<3.0$ & $3.0_{-1.6}^{+2.8}$ & $<3.0$ & $3.0_{-1.6}^{+2.2 .9}$ & $<1.00$ & $>0.7$ \\
\hline 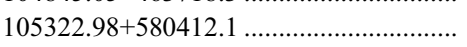 & $<3.0$ & $3.7_{-1.8}^{-3.6}$ & $<4.8$ & $4.3_{-2.0}^{+3.6}$ & $<1.30$ & $>0.5$ \\
\hline 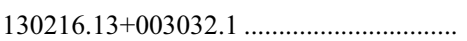 & $<3.0$ & $<4.8$ & $<4.8$ & $<6.4$ & $\ldots$ & $\ldots$ \\
\hline 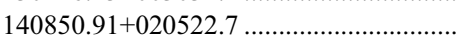 & $4.0_{-1.9}^{+3.2}$ & $26.2_{-51}^{+6.2}$ & $4.0_{-19}^{+3.2}$ & $30.0_{-54}^{+6.5}$ & $0.15_{-0.08}^{+0.13}$ & $2.4_{-0.6}^{+0.7}$ \\
\hline $141111.29+121737.4 \ldots \ldots \ldots \ldots \ldots \ldots \ldots \ldots \ldots \ldots \ldots$ & $<4.8$ & $10.9_{-3.3}^{+4.4}$ & $<6.4$ & $12.9_{-3.5}^{+4.7}$ & $<0.59$ & $>1.2$ \\
\hline $143352.20+022713.9$ & $<3.0$ & $<4.8$ & $<3.0$ & $<4.8$ & & \\
\hline 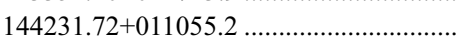 & $2.0_{-1.3}^{+2.7}$ & $36.7_{-6.0}^{+7.1}$ & $6.9_{-2.6}^{+3.8}$ & $43.6_{-6.6}^{+7.7}$ & $0.19_{-0.08}^{+0.11}$ & $2.0_{-0.4}^{+0.4}$ \\
\hline 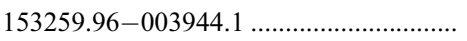 & $<3.0$ & $<3.0$ & $<3.0$ & $<3.0$ & & $\ldots$ \\
\hline 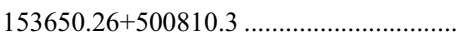 & $<3.0$ & $7.0_{-2.6}^{+3.8}$ & $8.0_{-2.8}^{+4.0}$ & $14.7_{-3.8}^{+4.9}$ & $1.15_{-0.78}^{+0.70}$ & $0.6_{-0.5}^{+1.0}$ \\
\hline 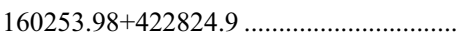 & $<4.8$ & $22.6_{-47}^{+5.8}$ & $2.9_{-16}^{+2.8}$ & $26.3_{-51}^{+6.2}$ & $0.13_{-0.08}^{+0.78}$ & $2.5_{-0.6}^{+0.8}$ \\
\hline 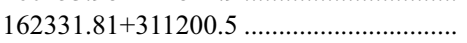 & $<3.0$ & $2.9_{-1.6}^{+2.9}$ & $<8.0$ & $5.7_{-2.3}^{+3.1 .5}$ & $<2.76$ & $>-0.2$ \\
\hline 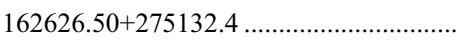 & $<3.0$ & $<4.8$ & $<3.0$ & $<4.8$ & & \\
\hline 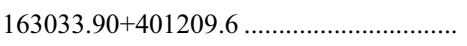 & $2.0_{-1.3}^{+2.7}$ & $13.6_{-3.6}^{+4.8}$ & $4.0_{-1.9}^{+3.2}$ & $17.4_{-4.1}^{+5.3}$ & $0.29_{-0.18}^{+0.25}$ & $1.8_{-0.6}^{+0.8}$ \\
\hline 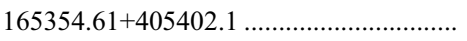 & $<3.0$ & $<3.0$ & $<6.4$ & $<6.4$ & $\ldots$ & $\ldots$ \\
\hline 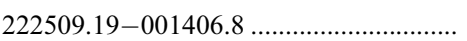 & $<3.0$ & $2.0_{-1.3}^{+2.7}$ & $<3.0$ & $2.0_{-1.3}^{+2.7}$ & $<1.50$ & $>0.2$ \\
\hline 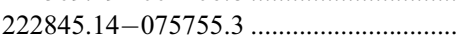 & $<3.0$ & $2.0_{-1.3}^{+2.3}$ & $<4.8$ & $3.0_{-1.6}^{+2.3}$ & $<2.40$ & $>-0.2$ \\
\hline
\end{tabular}

${ }^{a}$ Errors on the X-ray counts were computed according to Tables 1 and 2 of Gehrels (1986) and correspond to the $1 \sigma$ level; these were calculated using Poisson statistics. The upper limits are at the $95 \%$ confidence level and were computed according to Kraft et al. (1991). Upper limits of 3.0, 4.8, 6.4, and 8.0 indicate that $0,1,2$, and $3 \mathrm{X}$-ray counts, respectively, have been found within an extraction region of radius $1^{\prime \prime}$ centered on the optical position of the quasar (considering the background within this source-extraction region to be negligible).

${ }^{\mathrm{b}}$ We calculated errors at the $1 \sigma$ level for the band ratio (the ratio between the $2-8 \mathrm{keV}$ and $0.5-2 \mathrm{keV}$ bands) and effective photon index following the "numerical method" described in $\S 1.7 .3$ of Lyons (1991); this avoids the failure of the standard approximate-variance formula when the number of counts is small (see $\S 2.4 .5$ of Eadie et al. 1971). The photon indices have been obtained applying the correction required to account for the ACIS quantum-efficiency decay at low energy. 

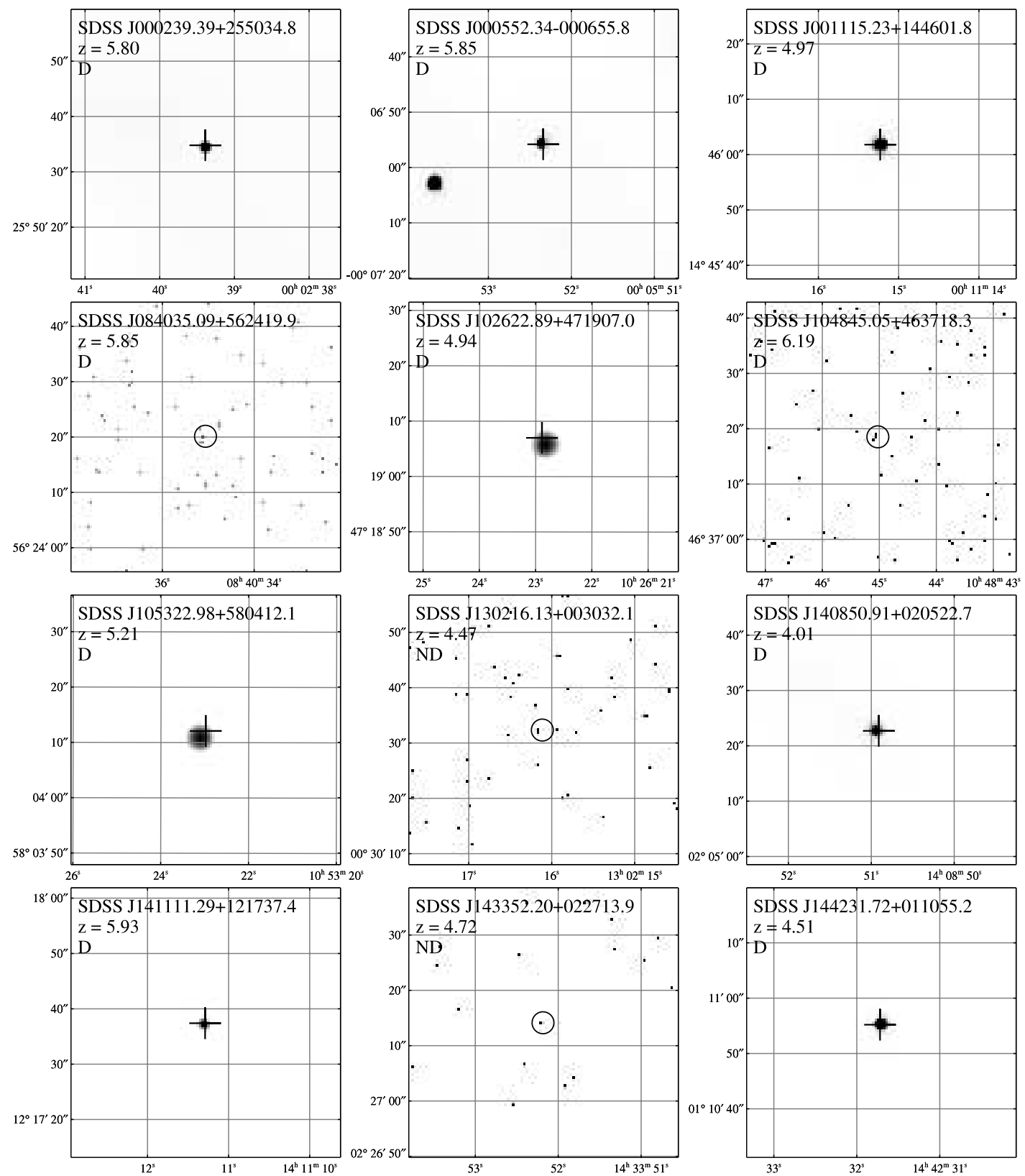

FIg. 2.-Full-band ( $0.5-8 \mathrm{keV})$ Chandra images of our quasars. Each panel spans $50^{\prime \prime} \times 50^{\prime \prime}$ on the sky; north is up, and east is to the left. The images of the sources with $>3$ counts have been adaptively smoothed at the $2 \sigma$ level; the optical positions of the quasars are marked by crosses. For sources with $\leq 3$ counts, the raw (unsmoothed) images and 2 " radius circles around the optical positions of the quasars are shown. A "D" ("ND") label in each panel indicates an X-ray detection (nondetection) of the source. Note that the image of SDSS J1532-0039 is a combination of our new Cycle 6 observation and an archival 5.09 ks Chandra exposure; the total exposure time for this image is $14.92 \mathrm{ks}$. The larger offsets between the optical and X-ray positions of the serendipitous sources, SDSS J1026+4719 and SDSS J1053+5804, are due to their large off-axis angles (see $\S 2$ ).

band $(0.5-8 \mathrm{keV})$ are reported in Table 2 . The counts were derived from manual aperture photometry, and they are consistent with the photometry obtained using wavdetect. Sixteen quasars were detected with 2-127 full-band counts; the largest number of counts was obtained from SDSS J0011+1446 which is a RLQ. The X-ray positions of the 14 targeted and detected sources differ by $0.2-0.7$ from their optical positions; the X-ray positions of the two serendipitous, off-axis detections differ by 0 "' $8-1$ ".'6 from their optical positions (Table 1). These results are consistent with the expected Chandra ACIS positional errors. We used the wavdetect tool a second time on the fields of the five undetected sources, with a false-positive probability threshold of $10^{-3}$, but none of the five sources was detected even at this lower significance level. Table 2 also includes the band ratio, calculated as the counts in the hard band divided by the counts in the soft band, and the effective power-law photon index $(\Gamma$, where $N_{E} \propto E^{-\Gamma} ; \Gamma$ was calculated from the band ratio using the Chandra PIMMS version 3.6a tool ${ }^{9}$ ) for each source. For the Cycle 4 and 6 sources the photon index was corrected for the quantum-efficiency decay of ACIS at low energies, caused by molecular contamination of the ACIS filters, using a time-dependent correction calculated with PIMMS.

Figure 2 presents the full-band Chandra images of the $50^{\prime \prime} \times 50^{\prime \prime}$ fields of all 21 quasars. The images of the 12 detected

9 See http://cxc.harvard.edu/toolkit/pimms.jsp. 


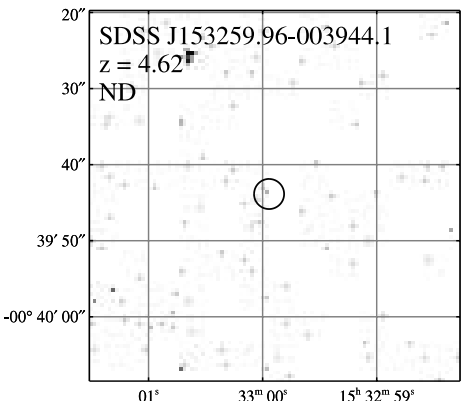

$01^{\mathrm{s}} \quad 33^{\mathrm{m}} 00^{\mathrm{s}} \quad 15^{\mathrm{h}} 32^{\mathrm{m}} 55^{\mathrm{s}}$
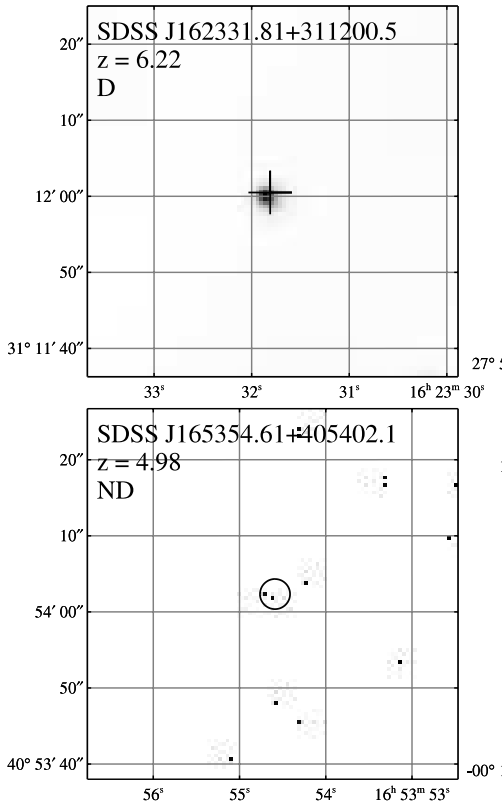
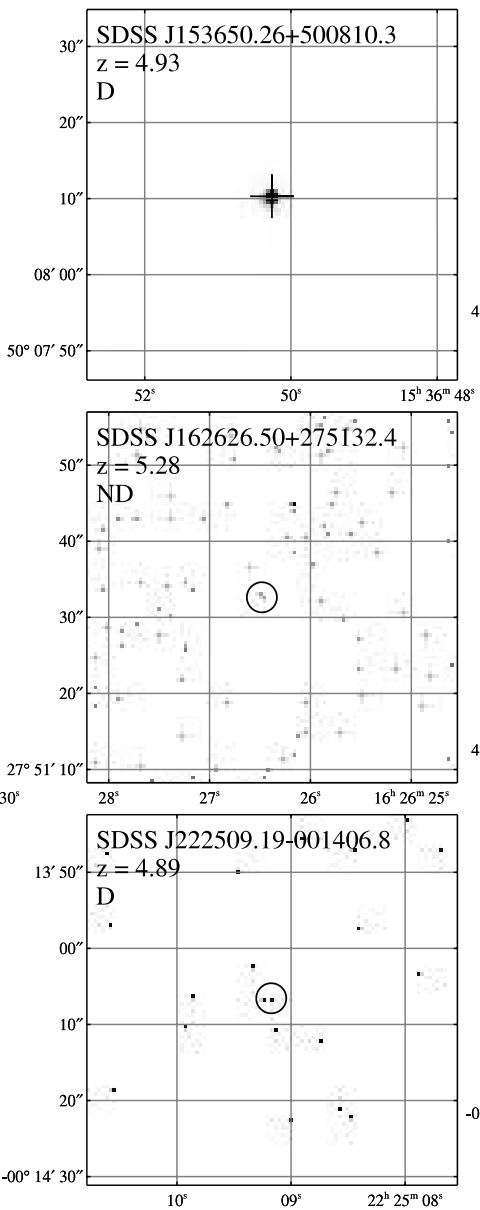

FIG. 2.-Continued
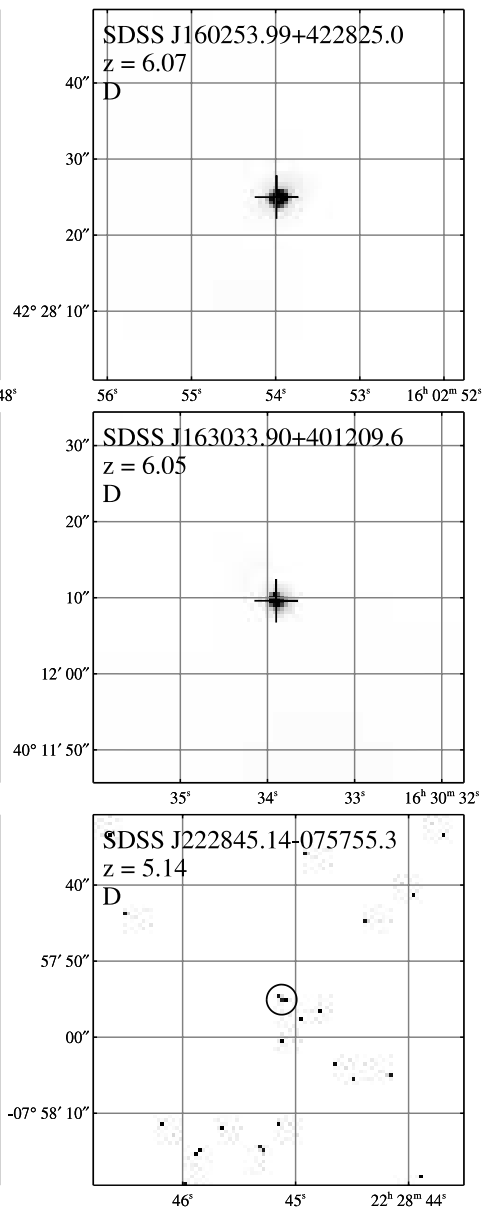

quasars with $>3$ counts were adaptively smoothed using the algorithm of Ebeling et al. (2006). The images of the four detected sources with $\leq 3$ counts, and the images of the fields of the five undetected quasars, were not smoothed; for these objects the raw images are shown. We note that the image of SDSS J1532-0039 was merged with an archival 5.09 ks Chandra exposure, centered on the optical position of the source, that did not provide an X-ray detection (Vignali et al. 2001); the merged image, which represents a total exposure time of $14.92 \mathrm{ks}$, is shown in Figure 2. Inspection of the Chandra images of our bright quasars does not reveal any excess X-ray emission in their vicinity that might be interpreted as gravitationally lensed images (e.g., Wyithe \& Loeb 2002; Richards et al. 2006) or jets (e.g., Schwartz 2002; Schwartz \& Virani 2004).

With the exception of SDSS J0005-0006, we did not find any significant excess of companions with respect to the cumulative number counts from X-ray surveys (e.g., Bauer et al. 2004) in the $\approx 300 \times 300 \mathrm{kpc}^{2}$ projected regions centered on the quasar positions; this is in agreement with previous X-ray searches around high- $z$ quasars (e.g., Vignali et al. 2005 and references therein). A bright X-ray source, with $\sim 60$ full-band counts, is apparent in the Chandra image of SDSS J0005-0006 with an angular distance of $\sim 20^{\prime \prime}$ from the target (Fig. 2). This source is classified as a galaxy in the SDSS DR4 catalog (Adelman-McCarthy et al. 2006), but due to its faintness $(i=22.1)$ an SDSS spectrum is not available. The X-ray to optical flux ratio of the source is just $\sim 3$ times lower than the relatively high X-ray to optical flux ratio of SDSS J0005-0006 (see Table 3), and based on photometry using co-added SDSS images in this area (Jiang et al. 2006) its photometric redshift is $z=0.575 \pm 0.175$ with an integrated probability of $63 \%$ (Weinstein et al. 2004). Given this redshift, we calculate an X-ray luminosity in the rest-frame $2-10 \mathrm{keV}$ band of $\approx 10^{43} \mathrm{ergs} \mathrm{s}^{-1}$ for this AGN candidate (the luminosity was obtained in the same way as for the quasars in our sample; see below).

Finally, we searched for rapid variability within the Chandra observations of our detected quasars by applying a KolmogorovSmirnov test to the photon arrival times, but no significant flux variations were detected. This is not surprising given the combination of the relatively short observations $(\lesssim 1 \mathrm{hr}$ in the rest frame) and the small number of detected photons from most of the sources.

\section{X-RAY, OPTICAL, AND RADIO PROPERTIES OF THE SAMPLE}

The main X-ray, optical, and radio properties of our sample are listed in Table 3:

Column (1).- The SDSS J2000.0 quasar coordinates, accurate to $\sim 00^{\prime \prime} 1$.

Column (2).- The Galactic column density in units of $10^{20} \mathrm{~cm}^{-2}$, taken from Dickey \& Lockman (1990) and obtained with the HEASARC $N_{\mathrm{H}}$ tool. $^{10}$

Column (3).- The monochromatic $\mathrm{AB}$ magnitude at a restframe wavelength of $1450 \AA\left(\mathrm{AB}_{1450}=-2.5 \log f_{1450 \AA}-48.6\right.$;

${ }^{10}$ See http://heasarc.gsfc.nasa.gov/cgi-bin/Tools/w3nh/w3nh.pl. 
TABLE 3

Optical, X-Ray, and Radio Properties

\begin{tabular}{|c|c|c|c|c|c|c|c|c|c|c|c|c|c|}
\hline $\begin{array}{c}\text { Object (SDSS J) } \\
\text { (1) }\end{array}$ & $\begin{array}{c}N_{\mathrm{H}}^{\mathrm{a}} \\
(2)\end{array}$ & $\begin{array}{c}\mathrm{AB}_{1450} \\
(3)\end{array}$ & $\begin{array}{l}M_{B} \\
\text { (4) }\end{array}$ & $\begin{array}{c}f_{2500 \AA^{\mathrm{b}}} \\
(5)\end{array}$ & $\begin{array}{c}\log \left(\nu L_{\nu}\right) \\
(2500 \AA) \\
(6)\end{array}$ & $\begin{array}{c}\text { Count Rate } \\
\text { (7) }\end{array}$ & $\begin{array}{c}f_{\mathrm{X}}^{\mathrm{d}} \\
(8)\end{array}$ & $\begin{array}{l}f_{2 \mathrm{keV}}^{\mathrm{e}} \\
\quad(9)\end{array}$ & $\begin{array}{c}\log \left(\nu L_{\nu}\right) \\
(2 \mathrm{keV}) \\
(10)\end{array}$ & $\begin{array}{c}\log L \\
(2-10 \mathrm{keV}) \\
(11)\end{array}$ & $\begin{array}{l}\alpha_{\mathrm{ox}} \\
(12)\end{array}$ & $\begin{array}{l}\Delta \alpha_{\mathrm{ox}}(\sigma)^{\mathrm{f}} \\
\quad(13)\end{array}$ & $\begin{array}{c}R^{\mathrm{g}} \\
(14)\end{array}$ \\
\hline $000239.39+255034.8 \ldots \ldots \ldots$. & 4.09 & $19.0^{\mathrm{h}}$ & -28.0 & 11.8 & 46.9 & $0.85_{-0.37}^{+0.58}$ & $3.8_{-1.7}^{+2.5}$ & 3.86 & 45.0 & 45.2 & $-1.72_{-0.10}^{+0.09}$ & $-0.00(0.0)$ & $<26.8^{\circ}$ \\
\hline 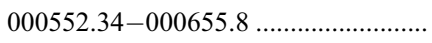 & 3.14 & $20.2^{\mathrm{h}}$ & -26.8 & 3.9 & 46.4 & $0.97_{-0.24}^{+0.30}$ & $4.2_{-1.1}^{+1.3}$ & 4.29 & 45.1 & 45.3 & $-1.52_{-0.05}^{+0.06}$ & $+0.14(0.9)$ & $<51.4$ \\
\hline $001115.23+144601.8 \ldots \ldots \ldots \ldots \ldots \ldots \ldots \ldots$ & 4.28 & $18.0^{\mathrm{i}}$ & -28.7 & 29.9 & 47.2 & $28.12_{-2.83}^{+3.14}$ & $97.1_{-9.8}^{+10.8}$ & 86.49 & 46.3 & 46.5 & $-1.36_{-0.03}^{+0.04}$ & $+0.40(3.1)$ & $135.9^{\circ}$ \\
\hline 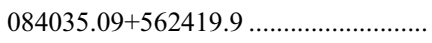 & 4.37 & $20.0^{\mathrm{j}}$ & -27.0 & 4.6 & 46.5 & $0.19_{-0.10}^{+0.19}$ & $0.8_{-0.4}^{+0.8}$ & 0.86 & 44.4 & 44.6 & $-1.81_{-0.12}^{+0.12}$ & $-0.15(1.0)$ & $<11.8$ \\
\hline 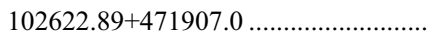 & 1.25 & $18.8^{\mathrm{i}}$ & -28.0 & 14.9 & 46.9 & $1.85_{-0.91}^{+1.55 \mathrm{~m}}$ & $8.9_{-4.4}^{+7.5}$ & 7.86 & 45.2 & 45.4 & $-1.64_{-0.12}^{+0.11}$ & $+0.08(0.5)$ & $<3.0$ \\
\hline $104845.05+463718.3 \ldots \ldots \ldots \ldots \ldots \ldots \ldots \ldots$ & 1.26 & $19.3^{\mathrm{k}}$ & -27.9 & 9.5 & 46.8 & $0.20_{-0.11}^{+0.20}$ & $0.8_{-0.4}^{+0.8}$ & 0.86 & 44.4 & 44.6 & $-1.94_{-0.12}^{+0.12}$ & $-0.22(1.4)$ & $<6.0$ \\
\hline $105322.98+580412.1$ & 0.56 & $19.6^{\mathrm{i}}$ & -27.3 & 7.2 & 46.6 & $0.92_{-0.46}^{+0.78 m}$ & $4.3_{-2.2}^{+3.7}$ & 4.02 & 45.0 & 45.2 & $-1.63_{-0.12}^{+0.11}$ & $+0.05(0.3)$ & $<7.3$ \\
\hline 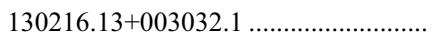 & 1.57 & $19.8^{\mathrm{i}}$ & -26.8 & 5.8 & 46.4 & $<0.44$ & $<1.4$ & $<1.14$ & $<44.3$ & $<44.5$ & $<-1.81$ & $<-0.16(>1.0)$ & $<8.3$ \\
\hline 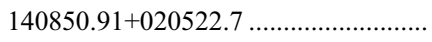 & 2.57 & $18.8^{\mathrm{i}}$ & -27.6 & 14.4 & 46.7 & $4.36_{-0.85}^{+1.03}$ & $18.5_{-3.6}^{+4.4}$ & 13.84 & 45.3 & 45.5 & $-1.54_{-0.04}^{+0.05}$ & $+0.16(1.0)$ & 8.1 \\
\hline 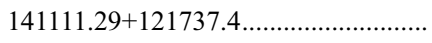 & 1.69 & $20.0^{\mathrm{h}}$ & -27.1 & 4.9 & 46.5 & $0.77_{-0.23}^{+0.31}$ & $3.2_{-1.0}^{+1.3}$ & 3.31 & 45.0 & 45.2 & $-1.60_{-0.07}^{+0.07}$ & $+0.07(0.5)$ & $<13.5$ \\
\hline $143352.20+022713.9 \ldots \ldots \ldots \ldots \ldots \ldots \ldots \ldots$ & 2.74 & $18.4^{\mathrm{i}}$ & -28.3 & 20.5 & 47.0 & $<1.37$ & $<4.5$ & $<3.84$ & $<44.9$ & $<45.1$ & $<-1.81$ & $<-0.08(>0.5)$ & $<2.4$ \\
\hline 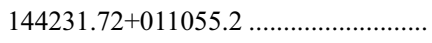 & 3.33 & $20.0^{\mathrm{i}}$ & -26.6 & 4.7 & 46.3 & $3.33_{-0.55}^{+0.65}$ & $11.2_{-1.9}^{+2.1}$ & 9.21 & 45.2 & 45.4 & $-1.42_{-0.04}^{+0.04}$ & $+0.22(1.4)$ & 31.7 \\
\hline $153259.96-003944.1 \ldots \ldots \ldots \ldots \ldots \ldots \ldots \ldots . .$. & 6.60 & $19.6^{1}$ & -27.0 & 6.9 & 46.5 & $<0.20^{\mathrm{n}}$ & $<0.9$ & $<0.79$ & $<44.2$ & $<44.4$ & $<-1.90$ & $<-0.23(>1.5)$ & $<1.0^{\mathrm{p}}$ \\
\hline 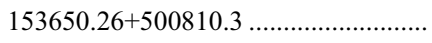 & 1.57 & $18.8^{\mathrm{i}}$ & -28.0 & 14.8 & 46.9 & $1.51_{-0.56}^{+0.82}$ & $6.2_{-2.3}^{+3.4}$ & 5.51 & 45.1 & 45.3 & $-1.70_{-0.08}^{+0.08}$ & $+0.02(0.1)$ & $<3.2$ \\
\hline $160253.98+422824.9$ & 1.33 & $19.9^{\mathrm{h}}$ & -27.2 & 5.4 & 46.6 & $1.71_{-0.36}^{+0.44}$ & $7.0_{-1.5}^{+1.8}$ & 7.39 & 45.3 & 45.5 & $-1.48_{-0.05}^{+0.05}$ & $+0.20(1.3)$ & $<10.3$ \\
\hline $162331.81+311200.5 \ldots \ldots \ldots \ldots \ldots \ldots \ldots \ldots . .$. & 2.05 & $20.1^{\mathrm{h}}$ & -27.0 & 4.2 & 46.5 & $0.17_{-0.09}^{+0.17}$ & $0.7_{-0.4}^{+0.7}$ & 0.75 & 44.3 & 44.5 & $-1.82_{-0.14}^{+0.12}$ & $-0.16(1.0)$ & $<12.3$ \\
\hline $162626.50+275132.4 \ldots \ldots \ldots \ldots \ldots \ldots \ldots \ldots \ldots$ & 3.27 & $18.5^{\mathrm{i}}$ & -28.4 & 19.3 & 47.0 & $<1.00$ & $<4.3$ & $<4.03$ & $<45.0$ & $<45.2$ & $<-1.80$ & $<-0.05(>0.4)$ & $<2.6$ \\
\hline $163033.90+401209.6 \ldots \ldots \ldots \ldots \ldots \ldots \ldots \ldots . .$. & 0.86 & $20.6^{\mathrm{k}}$ & -26.4 & 2.6 & 46.3 & $0.50_{-0.13}^{+0.17}$ & $2.0_{-0.5}^{+0.7}$ & 2.11 & 44.8 & 45.0 & $-1.57_{-0.05}^{+0.06}$ & $+0.06(0.4)$ & $<19.6$ \\
\hline $165354.61+405402.1$ & 1.94 & $19.3^{\mathrm{i}}$ & -27.5 & 9.4 & 46.7 & $<0.96$ & $<3.1$ & $<2.77$ & $<44.8$ & $<45.0$ & $<-1.74$ & $<-0.05(>0.3)$ & $<5.3$ \\
\hline $222509.19-001406.8 \ldots \ldots \ldots \ldots \ldots \ldots \ldots \ldots \ldots$ & 5.03 & $19.2^{\mathrm{i}}$ & -27.5 & 10.0 & 46.7 & $0.58_{-0.37}^{+0.77}$ & $2.1_{-1.4}^{+2.7}$ & 1.84 & 44.6 & 44.8 & $-1.82_{-0.18}^{+0.14}$ & $-0.12(0.8)$ & $<4.7$ \\
\hline 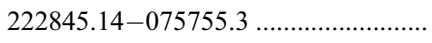 & 4.67 & $19.1^{\mathrm{i}}$ & -27.7 & 10.9 & 46.8 & $0.28_{-0.18}^{+0.38}$ & $1.0_{-0.7}^{+1.3}$ & 0.92 & 44.3 & 44.5 & $-1.95_{-0.20}^{+0.14}$ & $-0.24(1.6)$ & $<4.4$ \\
\hline
\end{tabular}

Neutral Galactic absorption column density in units of $10^{20} \mathrm{~cm}^{-2}$ taken from Dickey \& Lockman (1990).

Flux density at rest-frame $2500 \AA$ in units of $10^{-28} \mathrm{erg} \mathrm{cm}^{-2} \mathrm{~s}^{-1} \mathrm{~Hz}^{-1}$.

Observed count rate computed in the $0.5-2 \mathrm{keV}$ band in units of $10^{-3}$ counts $\mathrm{s}^{-1}$

${ }^{\mathrm{d}}$ Galactic absorption-corrected flux in the observed $0.5-2 \mathrm{keV}$ band in units of $10^{-15} \mathrm{erg} \mathrm{cm}^{-2} \mathrm{~s}^{-1}$.

${ }^{e}$ Flux density at rest-frame $2 \mathrm{keV}$ in units of $10^{-32} \mathrm{erg} \mathrm{cm}^{-2} \mathrm{~s}^{-1} \mathrm{~Hz}^{-1}$

${ }^{\mathrm{f}}$ The difference between measured and predicted $\alpha_{\mathrm{ox}}\left(\Delta \alpha_{\mathrm{ox}}\right)$, and the significance of that difference $(\sigma)$, based on the Steffen et al. (2006) $\alpha_{\mathrm{ox}}-L_{\nu}(2500 \AA)$ relation.

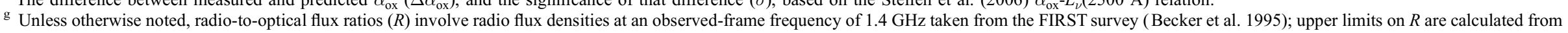
the $3 \sigma$ FIRST detection threshold at source position.

Taken from Fan et al. (2004).

Obtained from the SDSS spectrum, corrected for Galactic extinction (Schlegel et al. 1998) and fiber light loss (see $\S 3$ ).

Taken from Fan et al. (2006).

${ }^{k}$ Taken from Fan et al. (2003)

Estimated as $[(z+0.1)+(i-0.2)] / 2$ using the $i$ and $z$ magnitudes in Fan et al. (1999)

${ }^{m}$ Count rate corrected for the exposure map due to a large offset from the aim point (see $\S 2$ ).

${ }^{\mathrm{n}}$ Constraints on the count rate and subsequent X-ray parameters were obtained by including an additional Chandra exposure (see $\S 2$ ).

${ }^{\circ}$ Flux density at an observed-frame frequency of $1.4 \mathrm{GHz}$ taken from the NVSS survey (Condon et al. 1998); upper limits are calculated from the NVSS detection threshold of 2.5 mJy. 
Oke \& Gunn 1983). All the magnitudes have been corrected for Galactic extinction (Schlegel et al. 1998). In cases where the magnitudes were obtained from the SDSS spectra, a fiber lightloss correction has been applied; this was calculated as the difference between the synthetic $i$ magnitude (i.e., the integrated flux across the $i$ bandpass in the SDSS spectrum) and the photometric SDSS $i$ magnitude, assuming no flux variation between the photometric and spectroscopic epochs.

Column (4).- The absolute $B$-band magnitude, computed assuming a UV-optical power-law slope of $\alpha=-0.5\left(f_{\nu} \propto \nu^{\alpha}\right.$; Vanden Berk et al. 2001).

Columns (5) and (6).- The flux density and luminosity at a rest-frame wavelength of $2500 \AA$, computed from the magnitudes in column (3), assuming a UV-optical power-law slope of $\alpha=-0.5$.

Columns (7) and (8).- The count rate in the observed-frame $0.5-2 \mathrm{keV}$ band and the corresponding flux, corrected for Galactic absorption and the quantum-efficiency decay of Chandra ACIS at low energy (for the Cycle 4 and 6 sources). The fluxes have been calculated using PIMMS, assuming a power-law model with $\Gamma=2.0$, which is a typical photon index for luminous AGN with $0 \lesssim z \lesssim 6$ (e.g., Reeves \& Turner 2000; Page et al. 2005; Piconcelli et al. 2005; Shemmer et al. 2005; Vignali et al. 2005; see also $\S 5)$.

Columns (9) and (10).- The flux density and luminosity at a rest-frame energy of $2 \mathrm{keV}$, computed assuming $\Gamma=2.0$ and corrected for the quantum-efficiency decay of Chandra ACIS at low energy (for the Cycle 4 and 6 sources).

Column (11).- The luminosity in the rest-frame $2-10 \mathrm{keV}$ band.

Column (12).- The optical-X-ray power-law slope, $\alpha_{\mathrm{ox}}$, defined as

$$
\alpha_{\mathrm{ox}}=\frac{\log \left(f_{2 \mathrm{keV}} / f_{2500 \AA}\right)}{\log \left(\nu_{2 \mathrm{keV}} / \nu_{2500 \AA}\right)},
$$

where $f_{2 \mathrm{kev}}$ and $f_{2500 \AA}$ are the flux densities at rest-frame $2 \mathrm{keV}$ and $2500 \AA$, respectively. The errors on $\alpha_{\text {ox }}$ were computed following the "numerical method" described in $\S 1.7 .3$ of Lyons (1991), taking into account the uncertainties in the X-ray count rates and photon indices $(\Gamma=2.0 \pm 0.1)$, and the effects of possible changes in the UV-optical slope (from $\alpha=-0.5$ to $\alpha=-0.79)$.

Column (13).- The difference between the measured $\alpha_{\mathrm{ox}}$ (from col. [12]) and the predicted $\alpha_{\mathrm{ox}}$, given the UV luminosity from column (6), based on the $\alpha_{\mathrm{ox}}-L_{\nu}(2500 \AA)$ relation (given as eq. [2] of Steffen et al. 2006; see also Strateva et al. 2005). The statistical significance of this difference is also given in units of $\sigma$, where $\sigma=0.146$ for $31<\log L_{\nu}(2500 \AA)<32$, and $\sigma=0.131$ for $32<\log L_{\nu}(2500 \AA)<33$ (see Table 5 of Steffen et al. 2006).

Column (14).- The radio-loudness parameter (e.g., Kellermann et al. 1989), defined as $R=f_{5 \mathrm{GHz}} / f_{4400} \AA$ (the ratio of flux densities at rest-frame frequencies). The flux density at a rest-frame frequency of $5 \mathrm{GHz}$ was computed from either the FIRST (Faint Images of the Radio Sky at Twenty cm; Becker et al. 1995) or NVSS (NRAO VLA Sky Survey; Condon et al. 1998) flux densities at an observed-frame frequency of $1.4 \mathrm{GHz}$, assuming a radio power-law slope of $\alpha=-0.8$. Upper limits on $R$ are at the $3 \sigma$ level, since the positions of all our sources are known a priori. The flux densities at rest frame $4400 \AA$ were computed from the $\mathrm{AB}_{1450}$ magnitudes assuming a UV-optical power-law slope of $\alpha=-0.5$. Typical radio-loudness values are $>100$ for RLQs and $<10$ for radio-quiet quasars (RQQs). Most of our sources are RQQs. One source, SDSS J0011+1446, is a RLQ with $R=136$, and another source, SDSS J1442+0110, is radio moderate with $R=32$. Seven sources have upper limits on $R$ that are not tight enough to rule out the possibility that they might be radio moderate.

\section{NOTES ON INDIVIDUAL OBJECTS}

SDSS J001115.23+144601.8 $(z=4.97)$. - This is the only RLQ in our sample $(R=136)$, and it is one of the most X-rayluminous quasars at $z>4$ with $L_{2-10 \mathrm{keV}}=3.2 \times 10^{46} \mathrm{ergs} \mathrm{s}^{-1}$ (see Page et al. 2005; Vignali et al. 2003b, 2005). The basic $\mathrm{X}$-ray, optical, and radio properties of the source were first presented in Schneider et al. (2005). The $\Delta \alpha_{\text {ox }}(\sigma)=+0.40$ (3.1) of the source indicates that it significantly deviates from the $\alpha_{\mathrm{ox}^{-}}$ $L_{\nu}(2500 \AA)$ relation, being brighter by a factor of $\approx 11$ in X-rays than RQQs of matched UV luminosity. This suggests that the $\mathrm{X}$-ray emission from the source is jet-dominated. The relatively large number $(\approx 127)$ of photons detected from the source also allowed a basic investigation of its X-ray spectrum, which was extracted with the CIAO task psextract using a circular region of $2^{\prime \prime}$ radius centered on the X-ray centroid. The background region was an annulus with inner and outer radii of $5^{\prime \prime}$ and $25^{\prime \prime}$, respectively. The spectrum was binned in groups of 10 counts per bin. We used XSPEC version 11.3.2 (Arnaud 1996) to fit the spectrum with a power-law model and a Galactic-absorption component (Dickey \& Lockman 1990), which was kept fixed during the fit. The spectrum and its best-fit model and residuals appear in Figure 3. The best-fit photon index is $\Gamma=1.75_{-0.35}^{+0.37}$ with $\chi^{2}=11.02$ for 9 degrees of freedom; such a relatively flat power law is typical among RLQs (e.g., Reeves \& Turner 2000). Adding an intrinsic-absorption component at the redshift of the quasar did not improve the fit, and in particular did not remove the $2 \sigma$ residuals at $\approx 0.6$ and $\approx 1.2 \mathrm{keV}$. The residuals at $\approx 1.2 \mathrm{keV}$ are consistent in energy with a redshifted $\mathrm{Fe} \mathrm{K} \alpha$ line, but we do not expect such a line in a highly beamed and X-rayluminous source (e.g., Page et al. 2004). The remaining residuals in our spectrum are therefore difficult to interpret and should be investigated with a higher signal-to-noise ratio spectrum.

SDSS J104845.05+463718.3 $(z=6.19)$. - This is one of two BALQSOs in our sample. The quasar was discovered by Fan et al. (2003), who detected a hint of a Si IV BAL trough in the optical spectrum of the source. It was later confirmed as a BALQSO by Maiolino et al. (2004), based on its IR spectrum, and it is the most distant BALQSO discovered to date. Millimeter observations of this source detected dust emission and suggested a star formation rate of $\sim 2000 M_{\odot} \mathrm{yr}^{-1}$ (Bertoldi et al. 2003). BALQSOs typically show soft X-ray absorption with intrinsic $\mathrm{X}$-ray column densities in the range $N_{\mathrm{H}} \approx 10^{22}-10^{24} \mathrm{~cm}^{-2}$, rendering them weak X-ray sources (e.g., Gallagher et al. 1999, $2006)$. Since we have accessed highly penetrating $X$-rays $(\sim 4-$ $70 \mathrm{keV}$ ) from the source, any X-ray flux attenuation due to absorption at the quasar redshift has been mitigated, allowing easier detection. We find that the source has $\alpha_{\text {ox }}=-1.94$, which is consistent with the predicted value for a non-BAL RQQ with matched UV luminosity $\left(\alpha_{\mathrm{ox}}=-1.72\right)$ at a level of $1.4 \sigma$ (Table 3). The $\Delta \alpha_{\mathrm{ox}}=-0.22$ for the source is also consistent with the mean difference between the absorption-corrected $\alpha_{\mathrm{ox}}$ and the predicted $\alpha_{\mathrm{ox}}$ values determined for a sample of 35 BALQSOs (see Fig. 2 of Gallagher et al. 2006).

SDSS J130216.13+003032.1 $(z=4.47)$. - This WLQ was first noted by Anderson et al. (2001) to have weak Ly $\alpha$ emission and an optical spectrum that is similar to the prototype quasar of this class, SDSS J1532-0039 (Fan et al. 1999). We did not detect this source in our Chandra observation; the upper limit on 


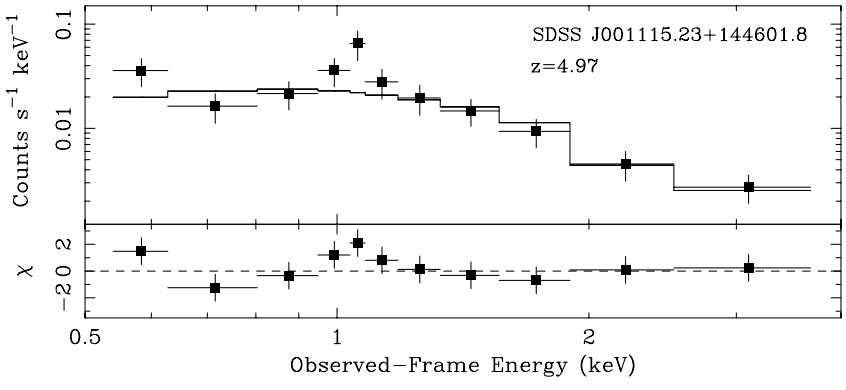

FIG. 3.-Chandra spectrum of SDSS J0011+1446. Filled squares represent the binned spectrum, and the solid histogram represents the best-fit power-law model. The $\chi$ residuals are in units of $\sigma$ with error bars of size 1 . Note the $2 \sigma$ residuals at $\sim 0.6$ and $\sim 1.2 \mathrm{keV}$.

its $\alpha_{\mathrm{ox}},-1.81$, is consistent at a level of $1 \sigma$ with the predicted $\alpha_{\text {ox }}$ (Table 3). Thus, the present data cannot prove that this source is anomalously X-ray weak.

SDSS J140850.91 + $020522.7(z=4.01)$. - This WLQ was first reported by Schneider et al. (2003). Even though it is one of three quasars in our sample that is radio-detected, its radioto-optical flux ratio, $R=8.1$, clearly classifies it as radio-quiet. It is one of two WLQs that we detect in the X-rays, and its $\alpha_{\mathrm{ox}}=-1.54$ is consistent with the predicted value at a level of $1 \sigma$ (Table 3).

SDSS J144231.72+011055.2 $(z=4.51)$. - This WLQ is radio moderate with $R=32$. It was first presented by Anderson et al. (2001), who noted its similarity to SDSS J1532-0039 (Fan et al. 1999). The source is quite X-ray bright, with $L_{2-10 \mathrm{keV}}=$ $2.5 \times 10^{45} \mathrm{ergs} \mathrm{s}^{-1}$. The relatively flat $\alpha_{\mathrm{ox}}=-1.42$ we find is typical of radio moderate-to-loud quasars, and it is consistent with the predicted $\alpha_{\mathrm{ox}}$ for RQQs of matched UV luminosity at a level of $1.4 \sigma$ (Table 3 ); this indicates a factor of $\sim 4$ excess in X-ray flux compared with the predicted RQQ value. The weak emission lines may be due to dilution by a beamed continuum source. The strong X-ray emission may also suggest the presence of a moderately beamed X-ray continuum component, which is in accordance with the fact that it is only moderately strong in the radio.

SDSS J153259.96-003944.1 $(z=4.62)$.- This is the prototype high- $z$ WLQ, discovered by Fan et al. (1999), who also found a radio-to-optical spectral index of $\alpha_{\text {ro }}>-0.02^{11}$ for the source; this $\alpha_{\text {ro }}$ lower limit is equivalent to $R<1.0$ (Table 3 ), making this source a RQQ. By monitoring the source during 2000-2001, Stalin \& Srianand (2005) found that it is optically variable, in the $R$ and $I$ bands, at a level of $\approx 0.3 \mathrm{mag}$; their claim of a $\sim 0.9$ mag difference between the SDSS photometry obtained in 1998 (Fan et al. 1999) and their 2001 photometry of the source was probably a consequence of incorrect magnitude conversions between the SDSS and Cousins magnitude systems. The source was targeted by Chandra in Cycle 2 but was undetected in a 5.09 ks observation (Vignali et al. 2001). We targeted this source with Chandra again in Cycle 6 and observed it for $9.83 \mathrm{ks}$. It remains undetected, and the combined exposure time of $14.92 \mathrm{ks}$ yields $\alpha_{\text {ox }}<-1.90$, which is consistent with the predicted $\alpha_{\text {ox }}$ at a level of $1.5 \sigma$ (Table 3). An additional Chandra exposure of $\approx 60 \mathrm{ks}$ would be required to determine whether this source is notably X-ray weak (at a $3 \sigma$ level).

SDSS J162331.81+311200.5 $(z=6.22)$.- -Discovered by Fan et al. (2004), this quasar has the strongest Ly $\alpha$ emission line

\footnotetext{
11 We adopt the definition of $\alpha_{\text {ro }}$ from Stocke et al. (1990); this is equivalent to the definition of $\alpha_{\text {ox }}$ (eq. [1]), where the $2 \mathrm{keV}$ flux density and frequency are replaced by the corresponding values at $5 \mathrm{GHz}$.
}

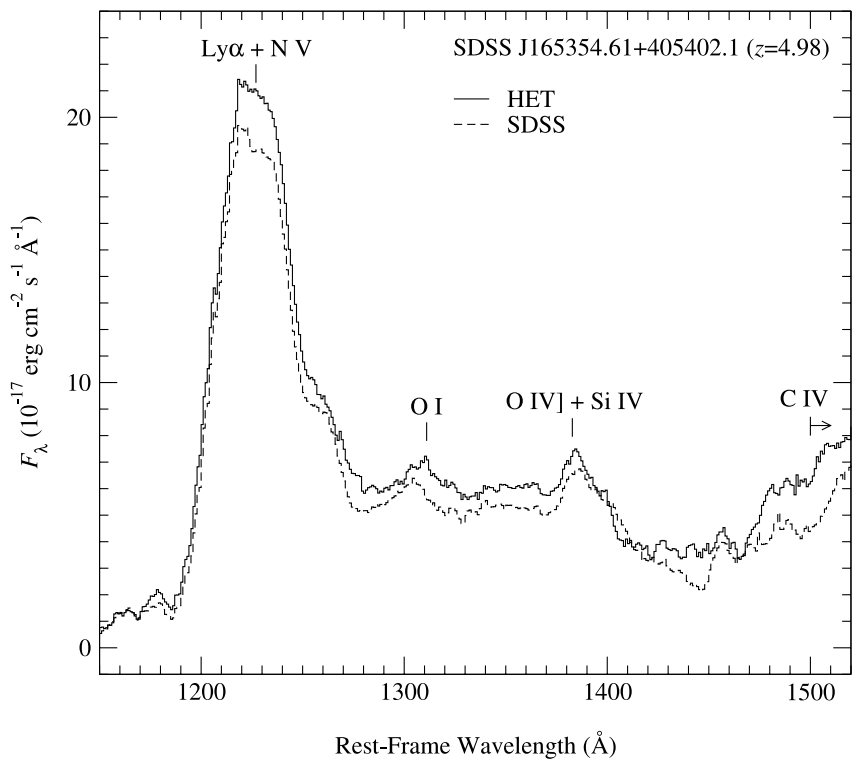

FIG. 4.-HET spectrum of SDSS J1653+4054 (solid curve) superposed on the SDSS spectrum of the source (dashed curve). Both spectra have been smoothed with a $10 \AA$ boxcar filter. A shallow C Iv BAL trough is apparent in both spectra at $\sim 1450 \AA$. The small differences between the two spectra, which are separated by 124 days in the rest frame, are probably due to systematics of the different instruments and calibration methods used.

known at $z>5$; the total rest-frame equivalent width (REW) of Ly $\alpha$ and $\mathrm{N} v \lambda 1240$ is $\gtrsim 150 \AA$, compared with a mean $\operatorname{REW}($ Ly $\alpha+\mathrm{N} v)$ of $69.3 \pm 18.0 \AA$ for $z>4$ quasars (Fan et al. 2001). Otherwise, this source is not particularly optically luminous; we detected it with Chandra and found $\alpha_{\mathrm{ox}}=-1.82$, which is consistent with the predicted value at a $1 \sigma$ level.

SDSS J165354.61+405402.1 $(z=4.98)$.- - Following the approval of our Chandra program, we reinvestigated the optical properties of our approved targets. The SDSS spectrum of this source (obtained on 2001 June 19) showed a hint of a C IV BAL trough, which we subsequently confirmed with a 15 minute exposure of the source, on 2003 July 1, using the Marcario LowResolution Spectrograph (Hill et al. 1998) on the $9 \mathrm{~m}$ Hobby-Eberly Telescope (HET; Ramsey et al. 1998; see Fig. 4). These spectra suggest that the quasar is a BALQSO with a C IV absorption trough having $\mathrm{REW} \simeq 25 \AA$ and an outflow velocity in the range $1.4 \times 10^{4} \lesssim v \lesssim 3.1 \times 10^{4} \mathrm{~km} \mathrm{~s}^{-1}$. We do not detect this source in our Chandra observation, obtaining $\alpha_{\mathrm{ox}}<-1.74$, which is consistent with the predicted value for a non-BAL RQQ with matched luminosity at a $0.3 \sigma$ level (Table 3). The $\Delta \alpha_{\text {ox }}<$ -0.05 we find is also consistent with the mean difference between the absorption-corrected $\alpha_{\mathrm{ox}}$ and the predicted $\alpha_{\mathrm{ox}}$ values determined for a sample of 35 BALQSOs (cf. Fig. 2 of Gallagher et al. 2006).

\section{X-RAY SPECTRAL PROPERTIES OF THE MOST DISTANT QUASARS}

Obtaining meaningful X-ray spectral properties, such as the power-law photon index and the intrinsic absorption column density, for the individual sources in this work (with the exception of SDSS J0011+1446) is hindered by the small numbers of detected photons. To date, such measurements have been done for only eight quasars at $z>4$ (e.g., Shemmer et al. 2005 and references therein). Our knowledge of the X-ray spectral properties of quasars at $z>4$ therefore relies mainly on joint spectral fitting of samples of such sources. For example, Vignali et al. (2005) 
TABLE 4

Properties of $z>5$ Radio-Quiet Quasars Included in the Joint Fitting

\begin{tabular}{|c|c|c|c|c|c|c|}
\hline $\begin{array}{l}\text { Object } \\
(\text { SDSS J) }\end{array}$ & $z$ & $N_{\mathrm{H}}^{\mathrm{a}}$ & X-Ray Observation Date & $\begin{array}{c}\text { Exposure Time }{ }^{\mathrm{b}} \\
(\mathrm{ks})\end{array}$ & Full-Band Total Counts & Reference \\
\hline $000239.39+255034.8 \ldots \ldots \ldots \ldots \ldots \ldots \ldots$ & 5.80 & 4.09 & 2005 Jan 24 & 5.87 & $5.0_{-2.2}^{+3.4}$ & 1 \\
\hline 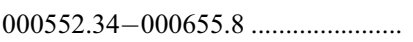 & 5.85 & 3.14 & $2005 \mathrm{Jul} 28$ & 16.93 & $17.6_{-4.1}^{+5.2}$ & 1 \\
\hline $023137.65-072854.5 \ldots \ldots \ldots \ldots \ldots \ldots \ldots \ldots \ldots \ldots \ldots \ldots \ldots$ & 5.41 & 2.90 & 2002 Sep 27 & 4.20 & $23.9_{-4.9}^{+6.0}$ & 2 \\
\hline $075618.14+410408.6 \ldots \ldots \ldots \ldots \ldots \ldots \ldots$ & 5.11 & 4.76 & 2002 Feb 8 & 7.33 & $14.8_{-3.8}^{+4.9}$ & 2 \\
\hline $083643.85+005453.3^{\mathrm{c}} \ldots \ldots \ldots \ldots \ldots \ldots \ldots$ & 5.77 & 4.40 & 2002 Jan 29 & 5.70 & $20.7_{-45}^{+5.6}$ & 3 \\
\hline $084035.09+562419.9 \ldots \ldots \ldots \ldots \ldots \ldots \ldots$ & 5.85 & 4.37 & 2005 Feb 3-4 & 15.84 & $3.0_{-1.6}^{+2.3}$ & 1 \\
\hline $103027.10+052455.0 \ldots \ldots \ldots \ldots \ldots \ldots \ldots \ldots \ldots \ldots \ldots \ldots$ & 6.28 & 2.70 & 2002 Jan 29 & 8.10 & $5.8_{-2.3}^{+3.6}$ & 3 \\
\hline $105322.98+580412.1 \ldots \ldots \ldots \ldots \ldots \ldots \ldots \ldots$ & 5.21 & 0.56 & 2001 July 5 & 4.70 & $4.3_{-2.0}^{+3.3}$ & 1 \\
\hline $120441.72-002149.5 \ldots \ldots \ldots \ldots \ldots \ldots \ldots$ & 5.03 & 2.12 & 2000 Dec 2 & 1.57 & $8.0_{-2.8}^{+4.0}$ & 4 \\
\hline $130608.26+035626.3 \ldots \ldots \ldots \ldots \ldots \ldots \ldots \ldots \ldots \ldots \ldots \ldots \ldots$ & 5.99 & 2.07 & 2002 Jan 29 & 8.20 & $16.8_{-4.1}^{+5.2}$ & 3 \\
\hline 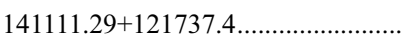 & 5.93 & 1.69 & 2005 Mar 17 & 14.27 & $12.9_{-3.5}^{+4.1}$ & 1 \\
\hline $160253.98+422824.9 \ldots \ldots \ldots \ldots \ldots \ldots \ldots \ldots$ & 6.07 & 1.33 & 2005 Oct 29 & 13.20 & $26.3_{-5.1}^{+6.2}$ & 1 \\
\hline $162331.81+311200.5 \ldots \ldots \ldots \ldots \ldots \ldots \ldots \ldots$ & 6.22 & 2.05 & 2004 Dec 29 & 17.21 & $5.7_{-2.3}^{+3.1}$ & 1 \\
\hline $163033.90+401209.6 \ldots \ldots \ldots \ldots \ldots \ldots \ldots \ldots \ldots \ldots \ldots \ldots \ldots$ & 6.05 & 0.86 & 2005 Nov 4 & 27.39 & $17.4_{-4.1}^{+5.3}$ & 1 \\
\hline $222845.14-075755.3 \ldots \ldots \ldots \ldots \ldots \ldots \ldots$ & 5.14 & 4.67 & 2003 May 5 & 7.04 & $3.0_{-1.6}^{+2.9}$ & 1 \\
\hline
\end{tabular}

a Neutral Galactic absorption column density in units of $10^{20} \mathrm{~cm}^{-2}$ taken from Dickey \& Lockman (1990).

b The Chandra exposure time has been corrected for detector dead time.

c This source has a radio counterpart in the FIRST catalog, with a flux of $1.11 \pm 0.15 \mathrm{mJy}$ at an observed-frame wavelength of $20 \mathrm{~cm}$; given the optical flux of the source, the radio-loudness parameter is $R \lesssim 10$, which ranks this quasar, at most, as radio moderate (e.g., Brandt et al. 2002).

REFERENCES.-(1) This work; (2) Vignali et al. (2003b); (3) Brandt et al. (2002); (4) Bechtold et al. (2003).

jointly fitted spectra of $48 z>4$ RQQs at a mean redshift of 4.3; they found a mean photon index of $\Gamma=1.93_{-0.09}^{+0.10}$ and have constrained the mean intrinsic neutral absorption column to $N_{\mathrm{H}} \lesssim 5 \times 10^{21} \mathrm{~cm}^{-2}$.

We jointly fitted the spectra of all optically selected $z>5$ RQQs, excluding BALQSOs, which were detected with $>2$ and $\lesssim 30$ full-band counts (the maximum limit prevents one or a few sources from dominating the average spectrum). This allows investigation of the mean X-ray spectral properties of the most distant quasars known, shedding light on the X-ray production mechanism and the central environment of quasars at the end of the reionization epoch. Using these criteria we found nine suitable sources from this work and six archival sources, all observed with Chandra. Table 4 lists all 15 sources used in the joint fitting process along with the total number of counts detected for each source. The mean (median) redshift of this group of quasars is 5.72 (5.85), with a total of 185 full-band counts, obtained in $158 \mathrm{ks}$.

The X-ray spectra were extracted with psextract using circular regions of $2^{\prime \prime}$ radius centered on the X-ray centroid of each source (except for SDSS J1053+5804, in which a 4 " radius was used due to its off-axis position on the CCD). Although the background is typically negligible in these Chandra observations, we have extracted background counts using annuli of different sizes (to avoid contamination from nearby X-ray sources) centered on each source. We used XSPEC v11.3.2 (Arnaud 1996) to fit the set of 15 unbinned spectra jointly with the following models: (1) a power-law model and a Galactic-absorption component (Dickey \& Lockman 1990), which was kept fixed during the fit, and (2) a model similar to the first with an added intrinsic (redshifted) neutral-absorption component. All fits assumed solar abundances (Anders \& Grevesse 1989; Dietrich et al. 2003), used the phabs absorption model in XSPEC with the Balucinska-Church \& McCammon (1992) cross-sections, and used the $C$-statistic (Cash 1979); this statistical approach allows one to retain all spectral information and to associate with each quasar its own Galactic-absorption column density and redshift. The errors associated with the best-fit X-ray spectral parameters are quoted at the $90 \%$ confidence level for one parameter of interest $(\Delta C=2.71$; Avni 1976; Cash 1979).

The joint-fitting process was carried out three times for both models. In the first run, we included the entire observed-energy range of all the spectra $(0.5-8 \mathrm{keV})$. In the second run we included only the common rest-frame energy range of all 15 sources: $3.64 \lesssim E_{\text {rest }} \lesssim 48.24 \mathrm{keV}$. In the third run we fitted the entire observed-energy range of 10 (out of 15) quasars with $z>5.5$ (the common rest-frame energy range for these quasars is almost identical to the entire observed-energy range due to their very narrow redshift distribution). Table 5 lists the best-fit parameters

TABLE 5

Best-Fit Parameters from Joint Fitting of $z>5$ Radio-Quiet Quasars

\begin{tabular}{|c|c|c|c|c|c|c|}
\hline Spectral Model & $\begin{array}{l}\text { Number of } \\
\text { Sources }\end{array}$ & $\begin{array}{c}\text { Mean (Median) } \\
\text { Redshift }\end{array}$ & Energy Range $^{\mathrm{a}}$ & $\begin{array}{c}N_{\mathrm{H}} \\
\left(10^{22} \mathrm{~cm}^{-2}\right)\end{array}$ & $\Gamma$ & $C$-Statistic (bins) \\
\hline Galactic absorbed power law. & 15 & $5.72(5.85)$ & E & 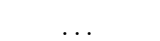 & $1.95_{-0.26}^{+0.27}$ & $158.6(175)$ \\
\hline 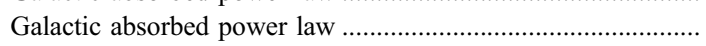 & 10 & $5.98(5.96)$ & $\mathrm{E}$ & . & $1.98_{-0.32}^{+0.31}$ & $121.2(124)$ \\
\hline Galactic absorbed power law and intrinsic absorption.............. & 15 & $5.72(5.85)$ & E & $\leq 5.98$ & $1.95_{-0.26}^{+0.30}$ & $158.6(175)$ \\
\hline Galactic absorbed power law and intrinsic absorption.............. & 15 & $5.72(5.85)$ & $\mathrm{C}$ & $\leq 6.63$ & $1.97_{-0.28}^{+0.30}$ & $154.8(168)$ \\
\hline
\end{tabular}

Notes. - The joint fitting process was carried out three times for each model. In the first run we fitted the entire energy range of all $z>5$ quasars, and in the second run we fitted their spectra in their common energy range. In the third run we fitted the spectra of $10 z>5.5$ quasars in their entire energy range.

${ }^{\mathrm{a}}$ The letter $\mathrm{E}$ stands for entire energy range of all the spectra, and $\mathrm{C}$ stands for the common rest-frame energy range among all sources (3.64-48.24 keV). 


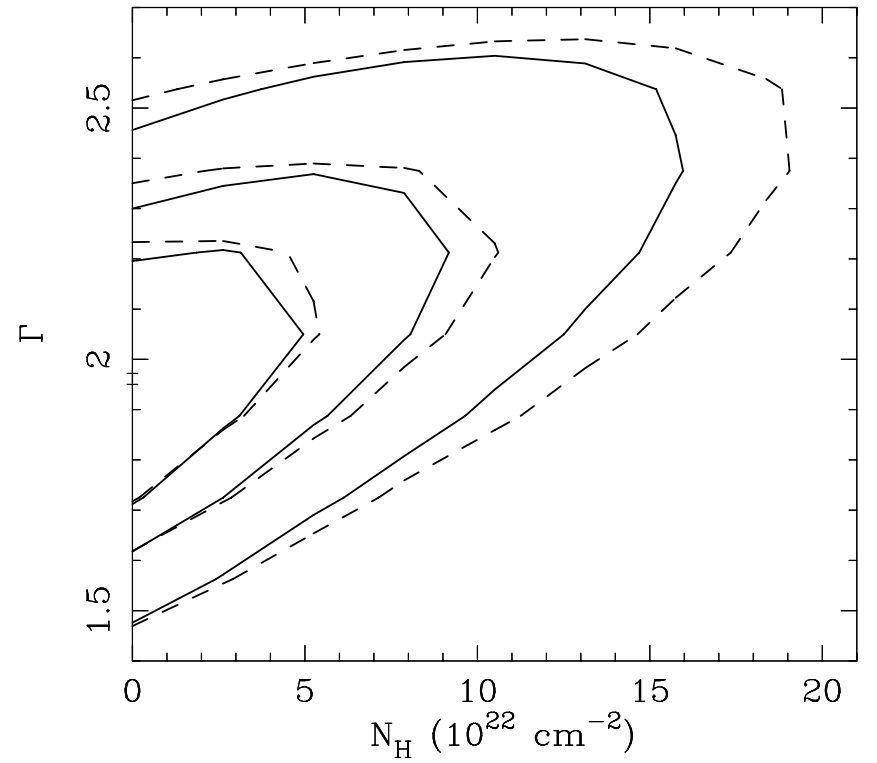

FIG. 5.-The $68 \%, 90 \%$, and $99 \%$ confidence regions for the photon index vs. intrinsic column density derived from joint spectral fitting of our sample of 15 RQQs at $z>5$. Solid (dashed) contours refer to the entire (common) energy range of the quasars (see $\S 5$ for more details). Note that the markers for the best-fit parameters in each fit lie on the vertical axis. from the joint-fitting process, and a contour plot of the $\Gamma-N_{\mathrm{H}}$ parameter space is shown in Figure 5. We find that, in the case of the entire energy range, the mean X-ray power-law photon index of $z>5$ RQQs is $\Gamma=1.95_{-0.26}^{+0.30}$, and their mean neutral intrinsic absorption column density is $N_{\mathrm{H}} \lesssim 6 \times 10^{22} \mathrm{~cm}^{-2}$. These results are consistent with those based on the common energy range and on the subgroup of $z>5.5$ quasars due to the relatively narrow redshift range of the sample and the fact that most of the quasars are at $z>5.5$.

The mean power-law photon index of optically selected $z>5$ RQQs is consistent with the mean photon index, $\Gamma=1.93_{-0.09}^{+0.10}$, obtained by jointly fitting spectra of 48 RQQs at $\langle z\rangle=4.3$ (Vignali et al. 2005), and with the mean photon index, $\Gamma=1.97_{-0.04}^{+0.06}$, obtained by jointly fitting high-quality spectra of eight RQQs at $\langle z\rangle=4.8$ (Shemmer et al. 2005). The mean photon index we obtain for $z>5$ RQQs is also consistent with photon-index measurements for individual RQQs at lower redshifts (e.g., Reeves \& Turner 2000; Vignali et al. 2001; Page et al. 2005; Piconcelli et al. 2005). Even though the upper limit we obtained on the mean neutral intrinsic absorption column in $z>5$ RQQs, $N_{\mathrm{H}} \lesssim 6 \times 10^{22} \mathrm{~cm}^{-2}$, is about an order of magnitude weaker than previous $N_{\mathrm{H}}$ upper limits obtained for $z>4$ RQQs with similar methods $\left(N_{\mathrm{H}} \lesssim 5 \times 10^{21} \mathrm{~cm}^{-2}\right.$ at $\langle z\rangle=4.3$; Vignali et al. 2005; $N_{\mathrm{H}} \lesssim 3 \times 10^{21} \mathrm{~cm}^{-2}$ at $\langle z\rangle=4.8$; Shemmer et al. 2005), it implies that RQQs were not heavily absorbed when the age of the universe was $\lesssim 1$ Gyr.

We have plotted $\alpha_{\text {ox }}$ for all the RQQs in our sample against their UV luminosity in Figure 6, and included all 333 RQQs

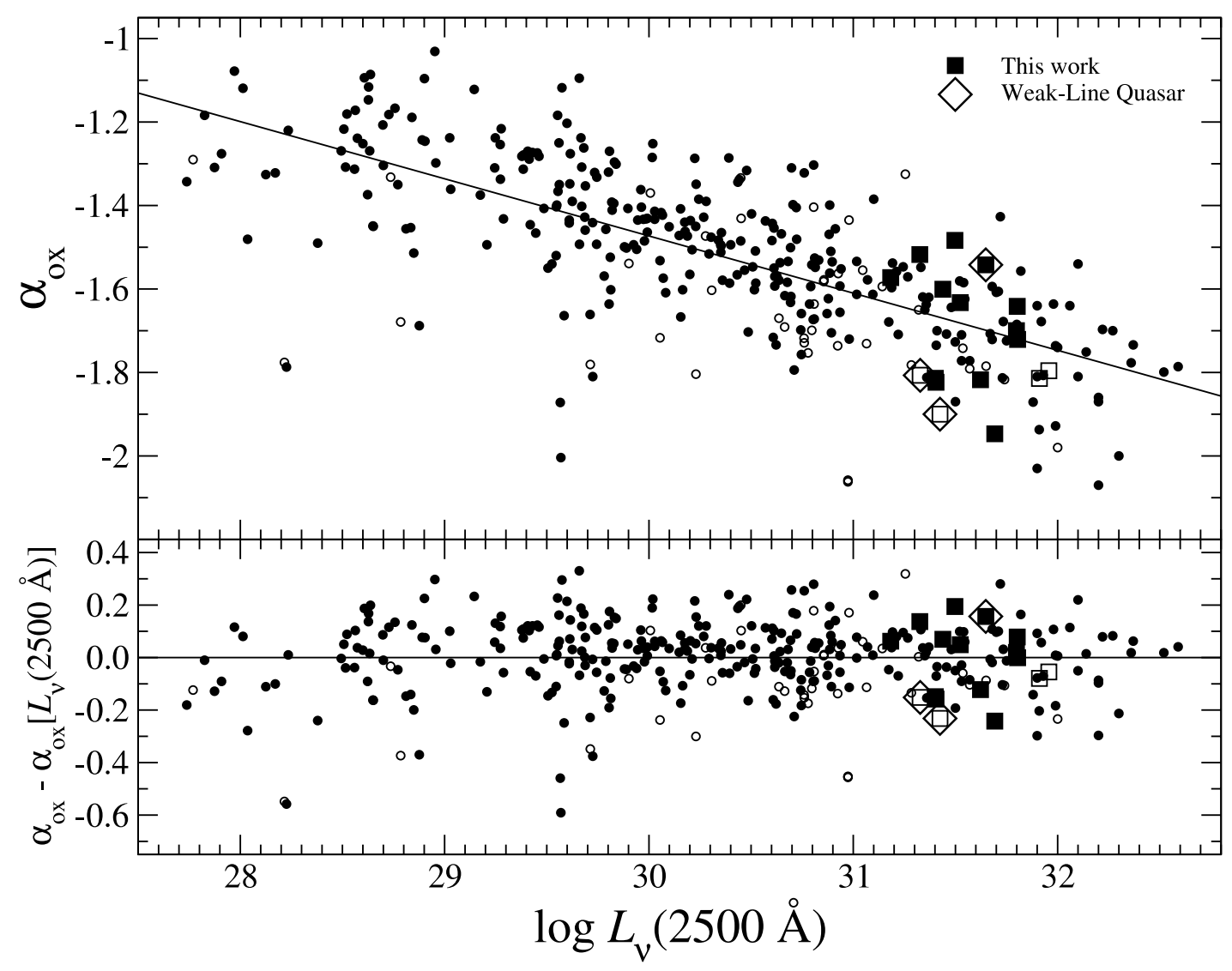

FIG. 6.-The $\alpha_{\text {ox }}-L_{\nu}(2500 \AA)$ relationship for RQQs, excluding known BALQSOs, adapted from Steffen et al. (2006). Open symbols indicate $\alpha_{\text {ox }}$ upper limits.

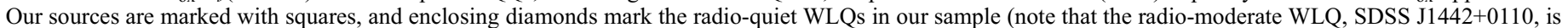

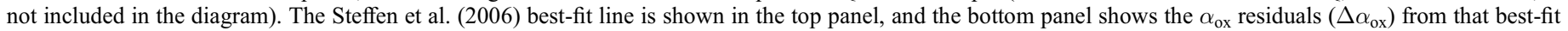
line. 


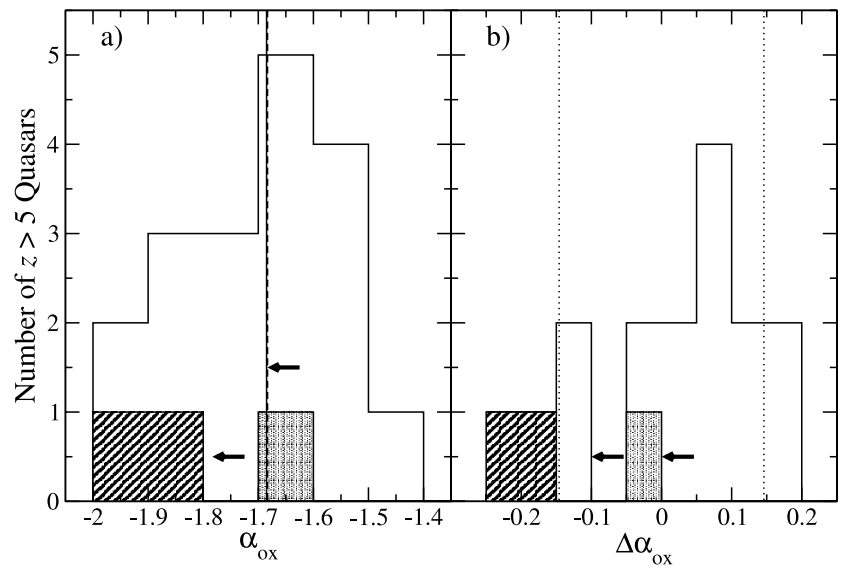

FIG. 7.- Histograms of (a) $\alpha_{\text {ox }}$ and (b) $\Delta \alpha_{\text {ox }}$ for all optically selected $z>5$ quasars from this work and the literature. Radio-loud quasars are shaded, and BALQSOs are hatched. Upper limits are marked by arrows. The solid (dashed) line in panel $a$ marks the mean measured (predicted) $\alpha_{\mathrm{ox}}$ of optically selected $z>5$ RQQs, excluding the BALQSOs (note that the two lines almost overlap). Dotted lines in panel $b$ mark the $1 \sigma$ range $(\sigma= \pm 0.146)$ for the distribution of $\Delta \alpha_{\text {ox }}$ for sources with $31<\log L_{\nu}(2500 \AA)<32$ from Steffen et al. (2006).

from the Steffen et al. (2006) study in this diagram. Our sources, which comprise most of the highest redshift quasars known, follow the correlation between $\alpha_{\mathrm{ox}}$ and UV luminosity with no detectable dependence on redshift. Figure 7 shows the $\alpha_{\text {ox }}$ distribution of 21 optically selected $z>5$ quasars (11 sources from this work and 10 from the literature) and the $\Delta \alpha_{\mathrm{ox}}$ distribution given their UV luminosities. We also calculated the mean $\alpha_{\text {ox }}$ of the 18 non-BAL RQQs in that sample using the ASURV software package (Lavalley et al. 1992) to account for two $\alpha_{\text {ox }}$ upper limits, and found $\left\langle\alpha_{\text {ox }}\right\rangle=-1.69 \pm 0.03$ (where the quoted error is the error on the mean). This value is consistent with the mean $\alpha_{\text {ox }}$ predicted from the Steffen et al. (2006) relation, $\left\langle\alpha_{\text {ox }}\right\rangle=$ $-1.68 \pm 0.15$ (where the quoted error is the standard deviation), given the mean UV luminosity of the sources. The $\Delta \alpha_{\text {ox }}$ distribution of $z>5$ quasars indicates that $\alpha_{\mathrm{ox}}$ values for most of the sources are consistent within $\pm 1 \sigma$ with the predicted values, and none of the sources is significantly X-ray weak (Fig. 7b). Our results strongly support the idea that AGN SEDs have not significantly evolved out to the highest observable redshifts.

\section{X-RAY CLUES TO THE NATURE OF WEAK EMISSION-LINE QUASARS}

We have observed four members of the rare class of WLQs with Chandra. Three of these sources are radio quiet, and one, SDSS J1442+0110, is radio moderate. SDSS J1408+0205 and SDSS $\mathrm{J} 1442+0110$ are the only X-ray-detected WLQs in our sample, and their $\alpha_{\text {ox }}$ values, -1.54 and -1.42 , respectively, appear relatively flat although not exceptional (Table 3). Both of these sources are also radio detected, although SDSS J1408+ 0205 is, by definition, a RQQ $(R=8.1)$. SDSS J004054.65$091526.8(z=4.98)$ is another radio-quiet X-ray-detected WLQ; it was serendipitously detected in an XMM-Newton observation of the galaxy cluster Abell 85, and its basic X-ray properties are presented in Schneider et al. (2003), who found $\alpha_{\mathrm{ox}}=$ -1.55 . Our data are therefore suggestive of two groups of WLQs: (1) radio-quiet WLQs that are also X-ray weak, including SDSS J1302+0030 and SDSS J1532-0039, and (2) WLQs that are mildly X-ray bright, with a relatively flat $\alpha_{\mathrm{ox}}$. The second group includes the two WLQs with radio detections (although these are not RLQs), SDSS J1408+0205 and SDSS J1442+0110, and the radio-undetected source SDSS J0040-0915.

Since the discovery of the prototype WLQ, SDSS J15320039 (Fan et al. 1999), about a dozen WLQs have been discovered at $z>2$ with $\operatorname{REW}(\operatorname{Ly} \alpha)<5 \AA$ (Anderson et al. 2001; Schneider et al. 2003, 2005; Collinge et al. 2005; Fan et al. 2006). We are also aware of at least two quasars at lower redshifts that lack high-ionization emission lines in their spectra, and whose optical luminosities are comparable to those of our WLQs; these are PG 1407+265 ( $z=0.94$; McDowell et al. 1995) and PHL 1811 ( $z=0.19$; Leighly et al. 2001) that may also be members of the WLQ class (see also Hawkins 2004). In contrast with their lack of Ly $\alpha$ and high-ionization lines, PG 1407+265 and PHL 1811 exhibit a strong and broad $\mathrm{H} \alpha$ and $\mathrm{H} \beta$ emission line, respectively. Another possible member of the WLQ class, 2QZ J215454.3-305654 at $z=0.494$, shows only an [O III] $\lambda 5007$ emission line, but since UV spectroscopy of the source was not yet carried out, it is unknown whether it displays higher ionization lines (Londish et al. 2004).

\subsection{Boosted Continuum versus Depleted High-Ionization Gas}

Some of the plausible explanations for the weakness, or absence, of high-ionization broad emission lines in WLQs include abnormal photoionizing continuum or broad emission line region (BELR) properties, and a low BELR covering factor. For example, such quasars may have a deficit of line-emitting gas in the vicinity of the central continuum source since an accretiondisk wind, proposed as a production site for some of the BELR lines (e.g., Murray \& Chiang 1997), may not form, perhaps due to exceptionally high Eddington ratios (e.g., Leighly et al. 2004), but see also Nicastro (2000). As a result of the high accretion rate, the continuum is expected to be correspondingly softer, thus suppressing high-ionization emission lines, but having little effect on the low-ionization species. A softer (X-ray) and bluer (UV-optical) continuum, as well as $\mathrm{H} \beta$ and $\mathrm{Fe}$ II blends are indeed observed in PHL 1811 (Leighly et al. 2004), although PG 1407+265 displays a "normal" quasar continuum (McDowell et al. 1995).

Many featureless-spectrum AGNs are identified as BL Lacertae objects (hereafter BL Lacs) whose emission lines are diluted by relativistic beaming of their optical continua (e.g., Urry \& Padovani 1995). Based on their weak-lined spectra alone, a BL Lac classification is another plausible explanation for the nature of WLQs. Most BL Lacs are low- to moderate-luminosity AGNs, discovered at $z \lesssim 1$ (e.g., Perlman et al. 2001 and references therein). By virtue of their weak-line spectra, the redshifts of BL Lacs are difficult to obtain and can be highly uncertain. Collinge et al. (2005) have found that BL Lac candidates with no significant proper motions and with either radio or X-ray detections tend to lie in specific regions of SDSS optical color-color diagrams. In particular, their $z<1$ BL Lac candidates have optical colors that are significantly redder than those of typical quasars. All of our WLQs have relatively accurate redshift measurements due to a clear Lyman break feature, and their optical spectra resemble those of six of the Collinge et al. (2005) BL Lac candidates at $z>2.15$ (the redshift threshold where the Lyman break feature enters the SDSS spectra).

Most known BL Lacs are strong radio and X-ray sources (relative to their optical emission), but the radio/X-ray-weak region in the BL Lac $\alpha_{\mathrm{ro}}-\alpha_{\text {ox }}$ "color-color" diagram is being increasingly populated by deep radio and X-ray surveys (e.g., Perlman et al. 2001 and references therein). In Figure 8 we plot the $\alpha_{\mathrm{ro}^{-}}$ $\alpha_{\text {ox }}$ diagram for all the BL Lacs from Perlman et al. (2001) and 


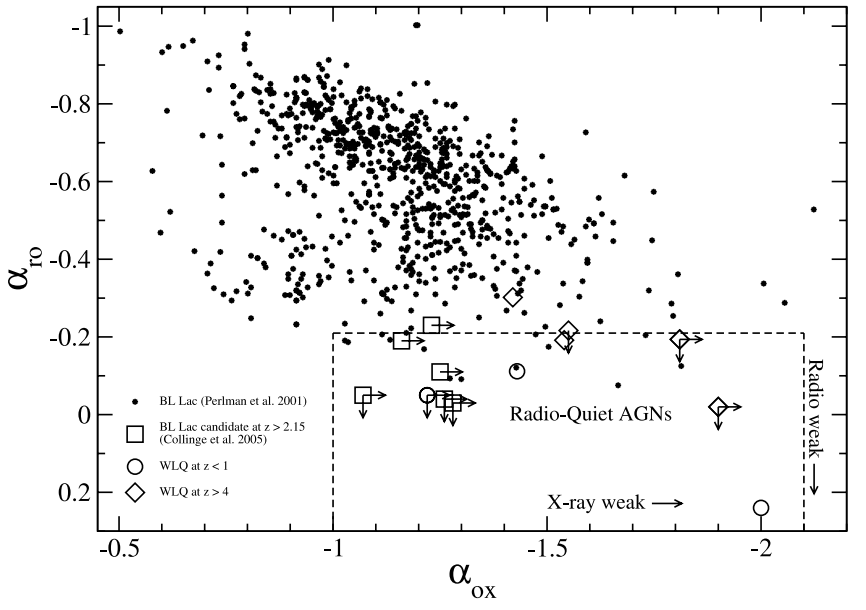

FIG. 8.-The $\alpha_{\mathrm{ro}}-\alpha_{\mathrm{ox}}$ diagram for BL Lacs, adapted from Perlman et al. (2001) and E. Perlman (2006, private communication). Stars mark BL Lacs selected from radio and X-ray surveys (Perlman et al. 2001). Squares mark $z>2.15$ BL Lac candidates from Collinge et al. (2005), circles mark the $z<1$ WLQs (2QZ J2154-3056, PG 1407+265, and PHL 1811; the first of these sources is not detected in both radio and X-rays, and the latter is the radioweakest source on this diagram), and diamonds mark $z>4$ WLQs. Right (down) pointing arrows mark $\alpha_{\text {ox }}\left(\alpha_{\text {ro }}\right)$ upper limits. Dashed lines mark the region containing most radio-quiet AGNs. Note that the WLQs and $z>2.15$ BL Lac candidates lie among known radio-weak BL Lacs, and that most sources from these three groups overlap with radio-quiet AGNs.

E. Perlman (2006, private communication); we also add to the plot the $z>2.15$ BL Lac candidates from Collinge et al. (2005), the three $z<1$ WLQs (2QZ J2154-3056, PG 1407+265, and PHL 1811), and the five $z>4$ WLQs discussed in this work. The region occupied by typical radio-quiet AGNs is also marked in Figure 8 (compare with Fig. 6). Apparently, the two radio- and $\mathrm{X}$-ray-detected $z>4 \mathrm{WLQs}$ (from our second group of WLQs), SDSS J1408+0205 and SDSS J1442+0110, are similar to (X-rayselected ) radio-weak BL Lacs. The two radio- and X-ray-undetected $z>4$ WLQs (from our first group of WLQs) and PHL 1811 might appear to constitute the tail of the radio/X-ray-weak BL Lac population, but their radio and X-ray properties are also consistent with those of typical RQQs. According to Fig. 8, most of the $z>2.15$ BL Lac candidates from Collinge et al. (2005) are consistent with the radio and X-ray properties of both radioweak BL Lacs and typical RQQs, although the loose constraints on their $\alpha_{\text {ox }}$ values (based on ROSAT measurements) prevent a proper distinction between BL Lacs and RQQs, as well as a comparison with the WLQs.

Since most BL Lacs at $z \lesssim 1$ are strong radio sources, it is interesting to note that most WLQs, and the Collinge et al. (2005) $z>2.15 \mathrm{BL}$ Lac candidates, are radio weak. The lack of radioloud ( $\alpha_{\text {ro }}<-0.4$, equivalent to $R>100$ ) WLQs might perhaps be attributed to the fact that they are all highly optically luminous sources. We investigated this possibility by examining the luminosities, $\alpha_{\text {ro }}$, and $\alpha_{\text {ox }}$ values of samples of lower redshift $(z \lesssim 1)$ BL Lacs. While BL Lacs from the EMSS sample (e.g., Rector et al. 2000) tend to be more radio/X-ray-weak at high optical luminosities, the BL Lacs from the larger RGB sample (LaurentMuehleisen et al. 1999) do not show this trend, and weak (and strong) radio sources are found at all luminosities. However, this is not sufficient to rule out a possible luminosity effect, since even the most luminous BL Lacs considered are 2 orders of magnitude less optically luminous than our WLQs, thus preventing a straightforward comparison of the radio properties between the two classes of sources. The lack of highly optically luminous BL Lacs may be a consequence of their selection, which is performed mainly with radio and X-ray surveys. Since it is possible that these selection methods have allowed highly optically luminous BL Lacs to escape detection, we checked for a radio-loudness-luminosity relation among the Collinge et al. (2005) optically selected sample of low-redshift BL Lac candidates, but no clear trend has been found.

Two other well-known characteristics of BL Lacs are rapid and large-amplitude optical flux variations, and high levels (up to $40 \%$ ) of optical polarization (e.g., Jannuzi et al. 1994). Stalin $\&$ Srianand (2005) have detected optical flux variations at a level of $\approx 0.3 \mathrm{mag}$ in the prototype WLQ, SDSS J1532-0039, on an observed-frame time baseline of 450 days ( 80 days in the rest frame), although this is somewhat lower than, and not as fast as, the level displayed by typical BL Lacs (e.g., Ghosh et al. 2000). Fan et al. (1999) have constrained the optical polarization level of SDSS J1532-0039 to be $\leq 4 \%$. This is consistent with singleepoch measurements of polarization levels in X-ray-selected BL Lacs, which are typically lower than the polarization levels observed in radio-selected BL Lacs (e.g., Jannuzi et al. 1994; Visvanathan \& Wills 1998). Therefore, low optical polarization levels in WLQs might be expected due to their radio weakness, regardless of whether they are BL Lacs or not.

While the nature of WLQs remains mysterious and apparently there is significant variety among this class of sources, a "boosted continuum" or a "suppression of high-ionization BELR lines" remain the most likely scenarios. The significant overlap in the radio and X-ray properties between different AGN classes (i.e., BL Lacs and radio-quiet AGNs), as well as the multiple selection biases, as depicted in the $\alpha_{\text {ro }}-\alpha_{\text {ox }}$ diagram (Fig. 8), might cause physically distinct AGN populations to blend together, and thus prevent a clear conclusion for the nature of WLQs. This calls for a more thorough and systematic investigation of the "continuum boosting" versus "high-ionization emission-line suppression" scenarios via more observations such as those described below, and detailed photoionization modeling. While relativistic boosting of the continuum may be consistent with the optical spectra, radio fluxes, and X-ray fluxes of the sources from our second group of WLQs, as well as with some of the $z>2.15 \mathrm{BL}$ Lac candidates of Collinge et al. (2005), it cannot hold for the two $z<1$ WLQs that display Balmer emission lines. It is also hard to accept a boosting scenario for our first group of WLQs and most of the $z>2.15$ BL Lac candidates of Collinge et al. (2005), due to the weakness of their radio and X-ray emission. In addition, the fact that radio-loud WLQs (with $R>100$ ) are missing is naturally explained by the "line suppression" scenario, which does not require that the quasars be radio-loud. The following observations for all $z>4$ WLQs and the $z>2.15$ BL Lac candidates of Collinge et al. (2005) are required to enable a clearer distinction between the two proposed scenarios:

1. Sensitive near-IR spectroscopy to reveal any low-ionization emission lines (e.g., Balmer or Fe II lines) and determine whether the UV-optical continua are typical of RQQs. Clear detection of low-ionization lines, and/or a typical (or bluer) continuum will support the "high-ionization line suppression" scenario, while the nondetection of such lines and redder continua will support the BL Lac interpretation.

2. Spitzer near-mid-IR observations (using IRAC and MIPS) may determine whether the SED is consistent with a typical AGN IR "bump" or with a beamed, "bumpless," continuum.

3. Optical-near-IR photometric monitoring to detect rapid and large-amplitude flux variations can check for a boosted continuum. 


\subsection{A Macro- or Micro-lensing Interpretation}

The absence of prominent optical emission lines in luminous, high-redshift quasars has raised several other possible explanations for their peculiar spectra. One of these is that high-redshift WLQs are not quasars at all, but rather normal galaxies (e.g., Ivison et al. 2005). Their measured luminosities are much larger than even the most luminous galaxies, but could perhaps be explained by magnification due to gravitational lensing. Our X-ray observations rule out that possibility, at least for the cases of the three X-ray-detected high-redshift WLQs that show AGN-like $\mathrm{X}$-ray properties, i.e., typical AGN X-ray-to-optical flux ratios (see the $\alpha_{\text {ox }}$ values in Table 3 and in Schneider et al. 2003; the $\mathrm{X}$-ray-to-optical flux ratios of typical galaxies are at least an order of magnitude lower than those of luminous AGNs; see, e.g., Kinney et al. 1996; Bauer et al. 2004).

Another possibility is that the continuum source in highredshift WLQs is amplified relative to the emission from the, much larger, broad emission-line region by gravitational microlensing, since the degree of amplification is inversely proportional to the size of the emitting region (e.g., Paczyński 1986; Schneider \& Weiss 1987; Ostriker \& Vietri 1990). Such microlensing events are expected to be both relatively short (monthsyears) and rare, and they might perhaps qualitatively explain the rarity of high-redshift WLQs among the quasar population (e.g., Wambsganss et al. 1990). Testing this scenario would require spectroscopic monitoring of our high-redshift WLQs to search for the reappearance of the emission lines. However, the SDSS images of our WLQs, and the X-ray images of our X-raydetected WLQs, do not show evidence for multiple images or lensing galaxies in their vicinity, and therefore a microlensing interpretation is unlikely (we also note that our WLQs were not targeted with the Hubble Space Telescope by Richards et al. 2006).

\section{SUMMARY}

We present new Chandra observations of $21 z>4$ quasars, including most of the highest redshift quasars known, as well as four members of the WLQ class. We have doubled the number of X-ray-detected quasars at $z>5$, allowing investigation of the $\mathrm{X}$-ray spectral properties of AGNs at the end of the reionization epoch. Our main results can be summarized as follows:

1. The mean power-law X-ray photon index of $z>5 \mathrm{RQQs}$ is $\Gamma=1.95_{-0.26}^{+0.30}$, and it is consistent with photon indices observed for RQQ samples at lower redshifts.

2. The upper limit on the mean intrinsic-absorption column density of $z>5$ RQQs is $N_{\mathrm{H}} \lesssim 6 \times 10^{22} \mathrm{~cm}^{-2}$, implying that RQQs at the dawn of the modern universe were not heavily absorbed.

3. The $\alpha_{\mathrm{ox}}$ values for individual RQQs in our sample, as well as the mean $\alpha_{\text {ox }}$ of $z>5$ RQQs, excluding BALQSOs, are con- sistent with the values predicted from their UV luminosities. This strengthens previous findings that AGN SEDs have not significantly evolved over cosmic time.

4. We detected two of our $z>4$ WLQs in X-rays, and distinguished two types of WLQs: radio-quiet WLQs that are also $\mathrm{X}$-ray-weak, and radio-quiet to radio-moderate WLQs (i.e., with $R<100$ ) that are mildly X-ray bright. Based on single-epoch optical spectra, as well as X-ray and radio fluxes of these sources, we discuss several possible interpretations for the weakness of the broad emission lines, and in particular, continuum boosting versus high-ionization emission-line suppression, which are the more likely scenarios. We propose sensitive near-IR spectroscopy, near-mid-IR photometry, and optical-near-IR monitoring to disentangle these two scenarios.

We gratefully acknowledge the financial support of Chandra X-ray Center grants GO3-4117X and GO5-6094X(O. S., W. N. B., and D. P. S), NASA LTSA grant NAG5-13035 (O. S., W. N. B., and D. P. S.), and NSF grants AST 03-07582 (D. P. S.), AST 03-07384 (X. F.), and AST 03-07409 (M. A. S.). We thank Pat Hall, Aaron Steffen, and Iskra Strateva for helpful comments and discussions, and Linhua Jiang for obtaining the photometric data for SDSS J0005-0006. We also thank Eric Perlman for kindly providing the $\alpha_{\text {ro }}$ and $\alpha_{\text {ox }}$ data for Figure 8. An anonymous referee is gratefully acknowledged for a prompt and constructive report that helped to improve the manuscript. Funding for the creation and distribution of the SDSS Archive has been provided by the Alfred P. Sloan Foundation, the Participating Institutions, the National Aeronautics and Space Administration, the National Science Foundation, the US Department of Energy, the Japanese Monbukagakusho, and the Max Planck Society. The SDSS Web site is http://www.sdss.org. The SDSS is managed by the Astrophysical Research Consortium (ARC) for the Participating Institutions. The Participating Institutions are the University of Chicago, Fermilab, the Institute for Advanced Study, the Japan Participation Group, The Johns Hopkins University, the Korean Scientist Group, Los Alamos National Laboratory, the Max-PlanckInstitute for Astronomy (MPIA), the Max-Planck-Institute for Astrophysics (MPA), New Mexico State University, University of Pittsburgh, University of Portsmouth, Princeton University, the United States Naval Observatory, and the University of Washington. The HET is a joint project of the University of Texas at Austin, the Pennsylvania State University, Stanford University, Ludwig-Maximillians-Universität München, and Georg-AugustUniversität Göttingen. The HET is named in honor of its principal benefactors, William P. Hobby and Robert E. Eberly. This research has made use of the NASA/IPAC Extragalactic Database (NED), which is operated by the Jet Propulsion Laboratory, California Institute of Technology, under contract with the National Aeronautics and Space Administration.

\section{REFERENCES}

Adelman-McCarthy, J. K., et al. 2006, ApJS, 162, 38

Anders, E., \& Grevesse, N. 1989, Geochim. Cosmochim. Acta, 53, 197

Anderson, S. F., et al. 2001, AJ, 122, 503

Arnaud, K. A. 1996, in ASP Conf. Ser. 101, Astronomical Data Analysis Software and Systems V, ed. G. H. Jacoby \& J. Barnes (San Francisco: ASP), 17

Avni, Y. 1976, ApJ, 210, 642

Balucinska-Church, M., \& McCammon, D. 1992, ApJ, 400, 699

Barger, A. J., Cowie, L. L., Brandt, W. N., Capak, P., Garmire, G. P., Hornschemeier, A. E., Steffen, A. T., \& Wehner, E. H. 2002, AJ, 124, 1839

Bassett, L. C., Brandt, W. N., Schneider, D. P., Vignali, C., Chartas, G., \& Garmire, G. P. 2004, AJ, 128, 523
Bauer, F. E., Alexander, D. M., Brandt, W. N., Schneider, D. P., Treister, E., Hornschemeier, A. E., \& Garmire, G. P. 2004, AJ, 128, 2048

Bechtold, J., et al. 2003, ApJ, 588, 119

Becker, R. H., White, R. L., \& Helfand, D. J. 1995, ApJ, 450, 559

Bertoldi, F., Carilli, C. L., Cox, P., Fan, X., Strauss, M. A., Beelen, A., Omont, A., \& Zylka, R. 2003, A\&A, 406, L55

Brandt, W. N., Guainazzi, M., Kaspi, S., Fan, X., Schneider, D. P., Strauss, M. A.,

Clavel, J., \& Gunn, J. E. 2001, AJ, 121, 591

Brandt, W. N., et al. 2002, ApJ, 569, L5

Carilli, C. L., et al. 2001, ApJ, 555, 625

Cash, W. 1979, ApJ, 228, 939

Collinge, M. J., et al. 2005, AJ, 129, 2542 
Condon, J. J., Cotton, W. D., Greisen, E. W., Yin, Q. F., Perley, R. A., Taylor, G. B., \& Broderick, J. J. 1998, AJ, 115, 1693

Dai, X., Chartas, G., Eracleous, M., \& Garmire, G. P. 2004, ApJ, 605, 45

Dickey, J. M., \& Lockman, F. J. 1990, ARA\&A, 28, 215

Dietrich, M., Hamann, F., Shields, J. C., Constantin, A., Heidt, J., Jäger, K., Vestergaard, M., \& Wagner, S. J. 2003, ApJ, 589, 722

Eadie, W. T., Dryard, D., James, F. E., Roos, M., \& Sadoulet, B. 1971, Statistical Methods in Experimental Physics (Amsterdam: North-Holland)

Ebeling, H., White, D. A., \& Rangarajan, F. V. N. 2006, MNRAS, 368, 65

Fan, X., et al. 1999, ApJ, 526, L57

2001, AJ, 121, 54

. 2003, AJ, 125,1649

- 2004, AJ, 128, 515

2006, AJ, 131, 1203

Farrah, D., Priddey, R., Wilman, R., Haehnelt, M., \& McMahon, R. 2004, ApJ, 611, L13

Freeman, P. E., Kashyap, V., Rosner, R., \& Lamb, D. Q. 2002, ApJS, 138, 185

Gallagher, S. C., Brandt, W. N., Chartas, G., Priddey, R., Garmire, G. P., \& Sambruna, R. M. 2006, ApJ, in press (astro-ph/0602558)

Gallagher, S. C., Brandt, W. N., Sambruna, R. M., Mathur, S., \& Yamasaki, N. 1999, ApJ, 519, 549

Garmire, G. P., Bautz, M. W., Ford, P. G., Nousek, J. A., \& Ricker, G. R. 2003, Proc. SPIE, 4851, 28

Gehrels, N. 1986, ApJ, 303, 336

Ghosh, K. K., Ramsey, B. D., Sadun, A. C., \& Soundararajaperumal, S. 2000, ApJS, 127, 11

Grupe, D., Mathur, S., Wilkes, B., \& Osmer, P. 2006, AJ, 131, 55

Hawkins, M. R. S. 2004, A\&A, 424, 519

Hill, G. J., Nicklas, H. E., MacQueen, P. J., Tejada, C., Cobos Duenas, F. J., \& Mitsch, W. 1998, Proc. SPIE, 3355, 375

Ivison, R. J., Smail, I., Bentz, M., Stevens, J. A., Menéndez-Delmestre, K., Chapman, S. C., \& Blain, A. W. 2005, MNRAS, 362, 535

Jannuzi, B. T., Smith, P. S., \& Elston, R. 1994, ApJ, 428, 130

Jiang, L., et al. 2006, AJ, in press (astro-ph/0602569)

Kaspi, S., Brandt, W. N., \& Schneider, D. P. 2000, AJ, 119, 2031

Kellermann, K. I., Sramek, R., Schmidt, M., Shaffer, D. B., \& Green, R. 1989, AJ, 98, 1195

Kinney, A. L., Calzetti, D., Bohlin, R. C., McQuade, K., Storchi-Bergmann, T., \& Schmitt, H. R. 1996, ApJ, 467, 38

Kraft, R. P., Burrows, D. N., \& Nousek, J. A. 1991, ApJ, 374, 344

Laurent-Muehleisen, S. A., Kollgaard, R. I., Feigelson, E. D., Brinkmann, W., \& Siebert, J. 1999, ApJ, 525, 127

Lavalley, M., Isobe, T., \& Feigelson, E. 1992, in ASP Conf. Ser. 25, Astronomical Data Analysis Software and Systems I, ed. D. M. Worrall, C. Biemesderfer, \& J. Barnes (San Francisco: ASP), 245

Leighly, K. M., Halpern, J. P., Helfand, D. J., Becker, R. H., \& Impey, C. D. 2001, AJ, 121, 2889

Leighly, K., Halpern, J., \& Jenkins, E. 2004, in ASP Conf. Ser. 311, in AGN Physics with the Sloan Digital Sky Survey, ed. G. T. Richards \& P. B. Hall (San Francisco: ASP), 277

Loeb, A., \& Barkana, R. 2001, ARA\&A, 39, 19

Londish, D., Heidt, J., Boyle, B. J., Croom, S. M., \& Kedziora-Chudczer, L. 2004, MNRAS, 352, 903

Lyons, L. 1991, Data Analysis for Physical Science Students (Cambridge: Cambridge Univ. Press)

Mahabal, A., Stern, D., Bogosavljević, M., Djorgovski, S. G., \& Thompson, D. 2005, ApJ, 634, L9

Maiolino, R., Oliva, E., Ghinassi, F., Pedani, M., Mannucci, F., Mujica, R., \& Juarez, Y. 2004, A\&A, 420, 889

Mathur, S., Wilkes, B. J., \& Ghosh, H. 2002, ApJ, 570, L5

McDowell, J. C., Canizares, C., Elvis, M., Lawrence, A., Markoff, S., Mathur, S., \& Wilkes, B. J. 1995, ApJ, 450, 585
Murray, N., \& Chiang, J. 1997, ApJ, 474, 91

Nicastro, F. 2000, ApJ, 530, L65

Oke, J. B., \& Gunn, J. E. 1983, ApJ, 266, 713

Ostriker, J. P., \& Vietri, M. 1990, Nature, 344, 45

Paczyński, B. 1986, ApJ, 301, 503

Page, K. L., O’Brien, P. T., Reeves, J. N., \& Turner, M. J. L. 2004, MNRAS, 347, 316

Page, K. L., Reeves, J. N., O’Brien, P. T., \& Turner, M. J. L. 2005, MNRAS, 364, 195

Pentericci, L., et al. 2003, A\&A, 410, 75

Perlman, E. S., Padovani, P., Landt, H., Stocke, J. T., Costamante, L., Rector, T., Giommi, P., \& Schachter, J. F. 2001, in ASP Conf. Ser. 227, Blazar Demographics and Physics, ed. P. Padovani \& C. Megan (San Francisco: ASP), 200

Petric, A. O., Carilli, C. L., Bertoldi, F., Fan, X., Cox, P., Strauss, M. A., Omont, A., \& Schneider, D. P. 2003, AJ, 126, 15

Piconcelli, E., Jimenez-Bailón, E., Guainazzi, M., Schartel, N. RodríguezPascual, P. M., \& Santos-Lleó, M. 2005, A\&A, 432, 15

Ramsey, L. W., et al. 1998, Proc. SPIE, 3352, 34

Rector, T. A., Stocke, J. T., Perlman, E. S., Morris, S. L., \& Gioia, I. M. 2000, AJ, 120, 1626

Reeves, J. N., \& Turner, M. J. L. 2000, MNRAS, 316, 234

Richards, G. T., et al. 2002, AJ, 123, 2945 2006, AJ, 131, 49

Schlegel, D. J., Finkbeiner, D. P., \& Davis, M. 1998, ApJ, 500, 525

Schneider, D. P., Schmidt, M., \& Gunn, J. E. 1991, AJ, 101, 2004

Schneider, D. P., et al. 2003, AJ, 126, 2579 2005, AJ, 130, 367

Schneider, P., \& Weiss, A. 1987, A\&A, 171, 49

Schwartz, D. A. 2002, ApJ, 571, L71

Schwartz, D. A., \& Virani, S. N. 2004, ApJ, 615, L21

Shemmer, O., Brandt, W. N., Vignali, C., Schneider, D. P., Fan, X., Richards, G. T., \& Strauss, M. A. 2005, ApJ, 630, 729

Spergel, D. N., et al. 2003, ApJS, 148, 175

Stalin, C. S., \& Srianand, R. 2005, MNRAS, 359, 1022

Steffen, A. T., Barger, A. J., Capak, P., Cowie, L. L., Mushotzky, R. F., \& Yang, Y. 2004, AJ, 128, 1483

Steffen, A. T., Strateva, I., Brandt, W. N., Alexander, D. M., Koekemoer, A. M., Lehmer, B. D., Schneider, D. P., \& Vignali, C. 2006, AJ, 131, 2826

Stern, D., Djorgovski, S. G., Perley, R. A., de Carvalho, R. R., \& Wall, J. V. 2000, AJ, 119,1526

Stocke, J. T., Morris, S. L., Gioia, I., Maccacaro, T., Schild, R. E., \& Wolter, A. 1990, ApJ, 348, 141

Strateva, I. V., Brandt, W. N., Schneider, D. P., Vanden Berk, D. G., \& Vignali, C. 2005, AJ, 130, 387

Urry, C. M., \& Padovani, P. 1995, PASP, 107, 803

Vanden Berk, D. E., et al. 2001, AJ, 122, 549

Vignali, C., Bauer, F. E., Alexander, D. M., Brandt, W. N., Hornschemeier, A. E., Schneider, D. P., \& Garmire, G. P. 2002, ApJ, 580, L105

Vignali, C., Brandt, W. N., Fan, X., Gunn, J. E., Kaspi, S., Schneider, D. P., \& Strauss, M. A. 2001, AJ, 122, 2143

Vignali, C., Brandt, W. N., Schneider, D. P., Garmire, G. P., \& Kaspi, S. 2003a, AJ, 125, 418

Vignali, C., Brandt, W. N., Schneider, D. P., \& Kaspi, S. 2005, AJ, 129, 2519

Vignali, C., et al. 2003b, AJ, 125, 2876

Visvanathan, N., \& Wills, B. J. 1998, AJ, 116, 2119

Wambsganss, J., Schneider, P., \& Paczynski, B. 1990, ApJ, 358, L33

Weinstein, M. A., et al. 2004, ApJS, 155, 243

Wyithe, J. S. B., \& Loeb, A. 2002, Nature, 417, 923

York, D. G., et al. 2000, AJ, 120, 1579 